\title{
On relations and homology of the Dehn quandle
}

\author{
JOEL ZABLOW
}

\begin{abstract}
Isotopy classes of circles on an orientable surface $F$ of genus $g$ form a quandle $Q$ under the operation of Dehn twisting about such circles. We derive certain fundamental relations in the Dehn quandle and then consider a homology theory based on this quandle. We show how certain types of relations in the quandle translate into cycles and homology representatives in this homology theory, and characterize a large family of 2-cycles representing homology elements. Finally we draw connections to Lefschetz fibrations, showing isomorphism classes of such fibrations over a disk correspond to quandle homology classes in dimension 2 , and discuss some further structures on the homology.
\end{abstract}

18G60, 57T99

\section{Relations}

\subsection{Introduction and definitions}

Throughout what follows, we shall be working on a closed orientable surface $F$, of genus $g$, which may bound a handlebody $H$. This handlebody will generally be thought of, in "standard form", as a series of $g$ holes arrayed consecutively along a loaf of bread. The words "circle" or "loop" will be considered, via abuse of notation, to mean isotopy class of circle (simple closed curve) in $F$, unless otherwise specified.

Let $P=\{$ isotopy classes of unoriented imbedded circles in $F$. We will let $G=$ $\operatorname{MCG}(F)$ denote the mapping class group of $F$.

Definition 1.1 A quandle consists of a group $G$ acting on the right on a set $S$, together with a pair of maps $\rceil, \Gamma: S \rightarrow G$ for which the following axioms hold:

(Q1) $a \bar{a} \mid=a \bar{a}=a$ for all $a \in S$

(Q2) $a|b \bar{b}|=a \bar{b} \mid \bar{b}=a$ for all $a, b \in S$

(Q3) $x \bar{b} \bar{a} \bar{b}=x \overline{a \bar{b}} \mid$ for all $a, b, x \in S$ "idempotence"

"inverses"

"conjugation" 
Note There are three other analogous variants of $(\mathrm{Q} 3)$ with differing combinations of the brackets $T$ and $\Gamma$.

These relations, in the given order, are analogs of the Reidemeister moves I, II, III, for knots and links. The definitions and notation for the quandle used here are taken from Kauffman [7], and will be used throughout. Quandles are also known as crossed $G$-sets or automorphic sets. The application of a bracket, right (\rceil$)$ or left $(\lceil)$, to an element $a \in S$, turns the element into an operator on other elements. Notice that the operating element is always written on the right of the operand. It is sometimes useful to write the quandle operations in a slightly different form. We write

$$
x *_{R} b \text { for } x \bar{b}
$$

and

$$
x *_{L} b \quad \text { for } x \bar{b} \text {. }
$$

Remark 1.2 The quandle operation is non-associative, but this is somewhat mitigated by the fact that axiom (Q3) is equivalent to a distributivity property in the quandle. Think of distributing a conjugation over a product of elements in a group. Using the notation above,

$$
\left(x *_{R} a\right) \bar{b}=x \bar{b}\left|*_{R} a \bar{b}\right|,
$$

for $a, b \in P$. Similar equations hold for the other combinations of brackets.

Definition 1.3 The algebraic object satisfying only the quandle relations (Q2) and (Q3) is called a rack (see Carter and Saito [1] or Fenn and Rourke [2]) or a crystal (see Kauffman [7]).

Examples 1.4 (1) Any group $G$ forms a quandle under the operation of conjugation, where for $a, g \in G$, we take $a \bar{g}=g a g^{-1}$ and $a \bar{g}=g^{-1} a g$.

(2) The set of $(n-1)$-dimensional planes in $\mathbb{R}^{n}$ forms a quandle, where both the operations $\bar{x} \mid$ and $\bar{x}$ correspond to reflection in the hyperplane $x$.

(3) The rack or crystal $C(K)$ associated to an oriented knot diagram $K$, is an invariant of regular isotopy (that is, only allowing Reidemeister moves II and III). The knot quandle is an ambient isotopy invariant [7].

Further discussion and more detailed examples of quandles, in particular finite quandles, may be found in [1] and [7].

Taking $P$ as mentioned above, to be the set of isotopy classes of imbedded circles in $F$, it was shown by the author [9] that the following holds: 
Proposition 1.5 The quintuple $\{P, \operatorname{MCG}(F), \mathbf{A}\rceil,\lceil\}$, has the structure of a quandle.

Here A: $G \rightarrow \operatorname{Aut}(P)$ denotes the action of $G=\operatorname{MCG}(F)$ on the set of circles $P$, and $\bar{a} \mid$ and $\bar{a}$ correspond respectively to right and left Dehn twists about the circle $a \in P$. So mnemonically,

$$
\begin{aligned}
\text { bracket on right } & =\text { right Dehn twist } \\
\text { bracket on left } & =\text { left Dehn twist. }
\end{aligned}
$$

Thus if $a$ and $b$ are circles in $F$ :

$$
\begin{aligned}
& b \bar{a}=\text { right Dehn twist about } a, \text { applied to } b \\
& b \bar{a}=\text { left Dehn twist about } a, \text { applied to } b
\end{aligned}
$$

For a given surface $F$, we call the quandle $Q$ just described, the Dehn quandle of $F$ (associated to the set of circles $P$ of $F$ ). Axiom (Q3) corresponds to the fact that we can conjugate a Dehn twist about one circle to a Dehn twist about another as seen schematically in Figure 1

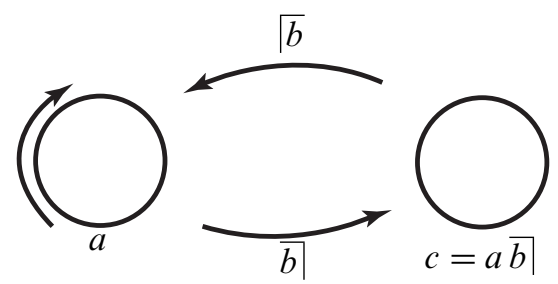

Figure 1: Conjugating twists

\subsection{Some relations}

For a given surface $F$, and the set of circles $P$ on $F$, we use the following notation hereafter. Let $x, y \in P$, such that the intersection of the circles representing the given isotopy classes, is minimal. Let

$$
|x \cap y|=\text { minimal geometric intersection number. }
$$

We now look at some relations in the Dehn quandle. These occur among circles which intersect a given circle once, and represent certain basic "symmetries" inherent in the Dehn quandle $Q$ of $F$. From Zablow [9], we have the following:

Proposition 1.6 (Fundamental relation F1) Let $a, b \in P$ such that $|a \cap b|=1$. Then $a \bar{b} \mid=b \bar{a}$. 
This relation underlies the similar, though more complicated relations to come. The proof is a homological argument in the standard homology $H_{1}(F)$. See Figure 2.

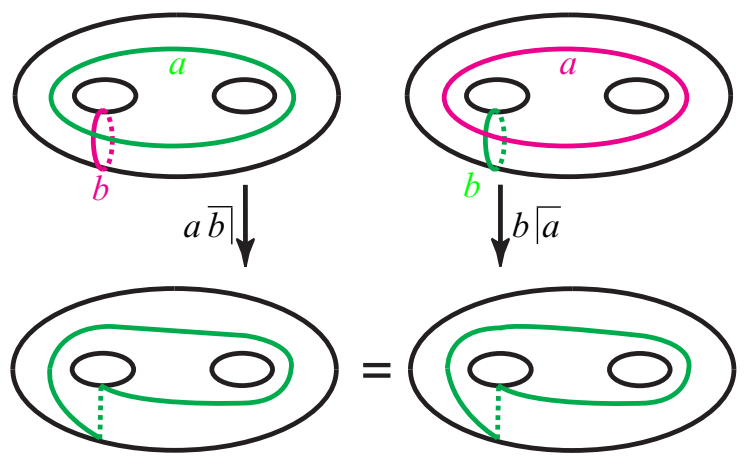

Figure 2: Fundamental relation F1

Here is a first consequence of this relation.

Theorem 1.7 (Relation F2) Let $a_{1}, a_{2}, c \in P$ such that $\left|a_{1} \cap c\right|=1,\left|a_{2} \cap c\right|=1$, and $\left|a_{1} \cap a_{2}\right|=0$. Then

$$
c \overline{a_{1}}\left|\overline{a_{2}}\right| \bar{c}\left|\overline{a_{1}}\right| \overline{a_{2}}=c
$$

\section{Proof}

$$
\begin{aligned}
& c \overline{a_{1}}\left|\overline{a_{2}}\right| \bar{c}\left|=a_{1} \sqrt{c} \overline{a_{2}}\right| \bar{c} \mid \quad \text { by fundamental relation with }\left|a_{1} \cap c\right|=1 \\
& =a_{1} \overline{a_{2}} \bar{c} \quad \text { by axiom }(\mathrm{Q} 3) \text { variant } \\
& =a_{1} \overline{c \mid a_{2}} \mid \quad \text { by fundamental relation with }\left|a_{2} \cap c\right|=1 \\
& =a_{1} \overline{a_{2}} \bar{c} \| \overline{a_{2}} \quad \text { by axiom }(\mathrm{Q} 3) \text { variant } \\
& =a_{1} \bar{c} \mid a_{2} \quad \text { since }\left|a_{1} \cap a_{2}\right|=0 \\
& =c \sqrt{a_{1}} \sqrt{a_{2}} \quad \text { by fundamental relation with }\left|a_{1} \cap c\right|=1
\end{aligned}
$$

Now apply $\overline{a_{2}} \mid \overline{a_{1}}$ on both sides. Use of (Q2) and the fact that $\overline{a_{2}}$ and $\overline{a_{1}}$ commute, since $\left|a_{1} \cap a_{2}\right|=0$, yields the desired equation.

There is a similar, though more involved relation with three mutually disjoint circles which each meet a fourth in a single point.

Theorem 1.8 (Relation F3) Let $a_{1}, a_{2}, a_{3}, c \in P$ such that $\left|a_{i} \cap a_{j}\right|=0$ for $i \neq j, 1 \leq i, j \leq 3$, and $\left|a_{i} \cap c\right|=1$ for $i=1, \ldots, 3$. Then

$$
c \overline{a_{1}}\left|\overline{a_{2}}\right| \overline{a_{3}}|\bar{c}| \overline{a_{1}}\left|\overline{a_{2}}\right| \overline{a_{3}}|\bar{c}| \overline{a_{1}}\left|\overline{a_{2}}\right| \overline{a_{3}}=c
$$


In the derivation below, let (F0) denote the commutativity relation among Dehn twists about non-intersecting circles. Also let (F1) denote the fundamental relation as mentioned above, and let (F2) denote the the relation $c \overline{a_{1}}\left|\overline{a_{2}}\right| \bar{c}\left|\overline{a_{1}}\right| \overline{a_{2}} \mid=c$, of the previous theorem. Recall that $(\mathrm{Q} 2)$ and $(\mathrm{Q} 3)$ denote the second and third quandle axioms. Brackets [...] or parentheses (...) highlight the "active" portions of the expressions, as we move from line to line.

Proof $c \overline{a_{1}}\left|\overline{a_{2}}\right| \overline{a_{3}}|\bar{c}| \overline{a_{1}}\left|\overline{a_{2}}\right| \overline{a_{3}}\left|\bar{c} \overline{a_{1}}\right| \overline{a_{2}} \mid \overline{a_{3}}$

$$
\begin{aligned}
& =\left(a_{1} \sqrt{c}\right) \overline{a_{2}}\left|\overline{a_{3}}\right| \bar{c}\left|\overline{a_{1}}\right| \overline{a_{2}}\left|\overline{a_{3}}\right| \bar{c}\left|\overline{a_{1}}\right| \overline{a_{2}} \mid \overline{a_{3}} \\
& =\left[\left(a_{2} \mid \overline{a_{1} \sqrt{c}}\right) \overline{a_{3}} \bar{c} \overline{a_{1}}\left|\overline{a_{2}}\right|\right] \overline{a_{3}}|\bar{c}| \overline{a_{1}}\left|\overline{a_{2}}\right| \overline{a_{3}} \\
& =\left[a_{3} \overline{a_{2} \sqrt{a_{1} \sqrt{c}}}\right] \bar{c}\left|\overline{a_{1}}\right| \overline{a_{2}}\left|\overline{a_{3}}\right| \bar{c}\left|\overline{a_{1}}\right| \overline{a_{2}} \mid \overline{a_{3}}
\end{aligned}
$$

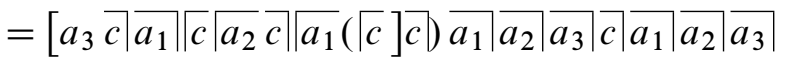

$$
\begin{aligned}
& =a_{3} \bar{c} \overline{a_{1}}|c| \overline{a_{2}} \bar{c}\left(\overline{a_{1}} \overline{a_{1}}\left|\overline{a_{2}}\right| \overline{a_{3}}|\bar{c}| \overline{a_{1}}\left|\overline{a_{2}}\right| \overline{a_{3}} \mid\right. \\
& =a_{3} \bar{c} \overline{a_{1} \mid}\left(\sqrt{c}\left|a_{2} \bar{c}\right| \overline{a_{2}}\left|\overline{a_{3}} \bar{c}\right| \overline{a_{1}}\left|\overline{a_{2}}\right| \overline{a_{3}}\right. \\
& =a_{3} \bar{c} \overline{a_{1}}\left(\mid \overline{a_{2} \bar{c}}\right) \overline{a_{2}}\left|\overline{a_{3}}\right| \bar{c}\left|\overline{a_{1}}\right| \overline{a_{2}} \mid \overline{a_{3}} \\
& =a_{3} \bar{c} \overline{a_{1}}\left(\mid \overline{c \mid \overline{a_{2}}}\right) \overline{a_{2}}\left|\overline{a_{3}}\right| \bar{c}\left|\overline{a_{1}}\right| \overline{a_{2}} \mid \overline{a_{3}} \\
& =a_{3} \bar{c} \overline{a_{1}}\left|\overline{a_{2}}\right| \bar{c}\left(\overline{a_{2}} \overline{a_{2}} \mid\right) \overline{a_{3}}\left|\bar{c} \overline{a_{1}}\right| \overline{a_{2}}\left|\overline{a_{3}}\right| \\
& =\left(a_{3} \bar{c}\left|\overline{a_{1}}\right| \overline{a_{2}}\left|c \overline{a_{3}}\right| \bar{c}\left|\overline{a_{1}}\right| \overline{a_{2}} \mid \overline{a_{3}}\right. \\
& =\left(c \overline{a_{3}}\right) \overline{a_{1}}\left|\overline{a_{2}}\right|\left[\bar{c} \overline{a_{3}}|\bar{c}|\right] \overline{a_{1}}\left|\overline{a_{2}}\right| \overline{a_{3}} \mid \\
& =c \overline{a_{1}}\left|\overline{a_{2}}\right|\left[\overline{a_{3}}\left|c \overline{a_{3}}\right|\right] \bar{c} \overline{a_{1}}\left|\overline{a_{2}}\right| \overline{a_{3}} \\
& =c \overline{a_{1}}\left|\overline{a_{2}}\right|\left[\overline{c \overline{a_{3}}} \mid\right] \bar{c}\left|\overline{a_{1}}\right| \overline{a_{2}} \mid \overline{a_{3}} \\
& =c \overline{a_{1}}\left|\overline{a_{2}}\left[\overline{a_{3} \sqrt{c}}\right] \bar{c}\right| \overline{a_{1}}\left|\overline{a_{2}}\right| \overline{a_{3}} \\
& \left.=c \overline{a_{1}} \overline{a_{2} \mid\left[\bar{c} \mid \overline{a_{3}}\right.}(\sqrt{c}] \bar{c} \mid\right) \overline{a_{1}}\left|\overline{a_{2}}\right| \overline{a_{3}} \\
& =c \overline{a_{1}}\left|\overline{a_{2}}\right| \bar{c}\left|a_{3} \overline{a_{1}}\right| \overline{a_{2}} \mid \overline{a_{3}} \\
& =c \overline{a_{1}}\left|\overline{a_{2}}\right| \bar{c} \overline{a_{1}}\left|\overline{a_{2}}\right| \overline{a_{3}} \overline{a_{3}} \\
& =c \overline{a_{1}}\left|\overline{a_{2}}\right| \bar{c}\left|\overline{a_{1}}\right| \overline{a_{2}} \\
& =c
\end{aligned}
$$

by (F1), $\left|a_{1} \cap c\right|=1$

by $(\mathrm{F} 1),\left|\left(a_{1} \bar{c}\right) \cap a_{2}\right|=1$

by (F1), $\left|a_{2} \sqrt{a_{1} \sqrt{c}} \cap a_{3}\right|=1$

by multiple (Q3)s

by $(\mathrm{Q} 2)$

by (Q2)

by (Q3)

by (F1), $\left|a_{2} \cap c\right|=1$

by (Q3)

by (Q2)

by (F1), $\left|a_{3} \cap c\right|=1$

by (F0), $\left|a_{3} \cap a_{1}\right|=0=\left|a_{3} \cap a_{2}\right|$

by (Q3)

by (F1), $\left|a_{3} \cap c\right|=1$

by (Q3)

by (Q2)

by (F0), $\left|a_{3} \cap a_{1}\right|=0=\left|a_{3} \cap a_{2}\right|$

by $(\mathrm{Q} 2)$

by (F2)

as required.

The braid relation in $\operatorname{MCG}(F)$ can be derived as a consequence of relation (F1) and axiom (Q3). See [9]. The relations (F1), (F2), and (F3) are based on certain symmetries 
of the action of $\operatorname{MCG}(F)$ on circles. Figure 5 below shows an example of relation (F3), and exhibits the underlying symmetry.

The family of relations of the type given by (F1), (F2), and (F3), obtained from a series of mutually disjoint circles $a_{i}$ which each intersect a circle $c$ once, does not extend to a similar relation (F4). In the descriptions of the configurations yielding the previous relations, the $a_{i}$ 's were mutually disjoint, but this does not preclude taking 2 or 3 copies of the same circle as $a_{i}$ in relations (F2) and (F3). Let us look at what this would entail for a relation (F4), following the pattern of (F2), (F3), if it exists, now using 4 copies of the same circle $a$. If the pattern continued, the relation would be

$c \overline{a_{1}}\left|\overline{a_{2}}\right| \overline{a_{3}}\left|\overline{a_{4}}\right| \bar{c}\left|\overline{a_{1}}\right| \overline{a_{2}}\left|\overline{a_{3}}\right| \overline{a_{4}}|\bar{c}| \overline{a_{1}}\left|\overline{a_{2}}\right| \overline{a_{3}}\left|\overline{a_{4}}\right| \bar{c}\left|\overline{a_{1}}\right| \overline{a_{2}}\left|\overline{a_{3}}\right| \overline{a_{4}}=c$

Consider the meridian $m$ and longitude $l$ of a torus. These intersect once and will play the roles of $a, c$ respectively. We look at a matrix representation of the action of $\operatorname{MCG}\left(T^{2}\right)$ on $H_{1}\left(T^{2}\right)$ with coefficients in $\mathbb{Z}$. We take the counterclockwise orientation on $l$ to be positive. Similarly, take a downward pointing arrow on $m$, on the front of the torus to be positive there. Let $\{m, l\}$ be the ordered basis for $H_{1}\left(T^{2}\right)$. If we continue to write the action of $\operatorname{MCG}\left(T^{2}\right)$ on the right of the operand circles, then we have the following correspondences:
(1) $m \Leftrightarrow\left(\begin{array}{l}1 \\ 0\end{array}\right)$
(2) $l \Leftrightarrow\left(\begin{array}{l}0 \\ 1\end{array}\right)$
(3) $\bar{m} \Leftrightarrow\left(\begin{array}{ll}1 & 0 \\ 1 & 1\end{array}\right)$
(4) $\bar{l} \Leftrightarrow\left(\begin{array}{cc}1 & -1 \\ 0 & 1\end{array}\right)$

A Mathematica computation for the corresponding matrices yields $\left(\begin{array}{c}-16 \\ 9\end{array}\right) \neq\left(\begin{array}{l}0 \\ 1\end{array}\right)$, so the pattern does not continue, and such an (F4) does not exist.

\section{Homology of the Dehn quandle and rack}

\subsection{Definitions and conventions}

In the sequel, we adopt many of the notations, definitions, and conventions regarding rack and quandle chain and homology groups from Carter and Saito [1]. For a quandle $Q$, let $C_{n}^{R}(Q)$ be the free abelian group generated by $n$-tuples $\left(x_{1}, \ldots, x_{n}\right)$ of elements of $Q$. This is the rack chain group. Such a tuple will be referred to as an 
$n$-simplex. Note that this differs from the conventional definition where a $k$-simplex involves $k+1$ terms. The boundary homomorphism $\partial_{n}: C_{n} \rightarrow C_{n-1}$ is defined by

$$
\begin{aligned}
\partial_{n}\left(x_{1}, \ldots, x_{n}\right)= & \sum_{i=2}^{n}(-1)^{i}\left[\left(x_{1}, x_{2}, \ldots, x_{i-1}, \hat{x}_{i}, x_{i+1}, \ldots, x_{n}\right)\right. \\
& \left.-\left(x_{1} \overline{x_{i}}, x_{2} \overline{x_{i}}, \ldots, x_{i-1} \overline{x_{i}}, x_{i+1}, \ldots, x_{n}\right)\right] .
\end{aligned}
$$

We take $\partial_{1}: C_{1} \rightarrow C_{0}$ to be the 0 homomorphism. $\left(C_{*}^{R}(Q), \partial\right)$ forms a chain complex. Following [1], an $n$-simplex which has consecutive terms $x_{i}=x_{i+1}$, where $1 \leq i \leq$ $n-1$, is degenerate. $C_{n}^{D}(Q)$ is the $n$th degenerate chain group. As a consequence of axiom (Q1), $\partial: C_{n}^{D}(Q) \rightarrow C_{n-1}^{D}(Q)$, and thus the chain complex of degenerate chains forms a subcomplex of the rack chain complex, $\left(C_{*}^{R}(Q), \partial\right)$. The quandle chain complex is then the quotient $C_{*}(Q)=C_{*}^{R}(Q) / C_{*}^{D}(Q)$ where degenerate simplices are considered to be 0 . By abuse of notation, we use $\partial$, defined as above, as the boundary operator in the quandle chain complex as well. Notationally, the rack and degenerate groups will be adorned with $R, D$ respectively, while the quandle groups are unadorned. The distinction between the rack and quandle chain groups, their subgroups and homology will become important later. Throughout the remainder of this article, we will assume that all the groups in question, in particular the quandle chain groups, $C_{n}(Q)$, cycle groups $Z_{n}(Q)$, boundary groups $B_{n}(Q)$, and homology groups, $H_{n}(Q)$, which arise from the quandle chain complex are taken with integer, $\mathbb{Z}$, coefficients unless otherwise stated, and $Q$ will be the Dehn quandle of a specified surface.

For the moment, we concentrate on the quandle chain complex and groups. Notice that this is a cubical chain complex, in which the boundary of an $n$-simplex has $2 n-2$ terms. For later arguments and discussion, it will be often be convenient to represent a 1-simplex as a point, 2-simplices as line segments, 3-simplices as square 2-cells, and 4-simplices as cubical 3-cells etc. Specifically, a 2-simplex will be given as an oriented segment, with the source endpoint being negative, and the target endpoint being positive. A 3-simplex will be given as a square oriented in a counterclockwise fashion, whose boundary terms are +--+ as given by the boundary formula. The orientations of those edges of the boundary corresponding to positive terms will be taken to agree with the overall orientation, and those corresponding to negative terms will be opposite the orientation of the square. Cancellation of a pair of identical simplices, having opposite signs, will be denoted by identifying the corresponding points, edges, or faces, of the appropriate simplices (simplex). The identifications will be made so that the orientations on the identified faces (edges) agree. 
In the case where a boundary edge or face of a simplex is 0 , this will be indicated pictorially by collapsing the appropriate edge or face. Thus a 3-simplex with a degenerate ( 0 ) edge in its boundary will be represented by a triangular 2-cell. Figure 3 illustrates the effect of the boundary operator on some distinct individual 3-simplices, and shows the use of the conventions mentioned above and the identifications (when they exist). So a general 3-chain can be represented as a surface comprised of such squares and triangles, identified along pairs of edges which represent the same 2-simplex but which occur with opposite signs.
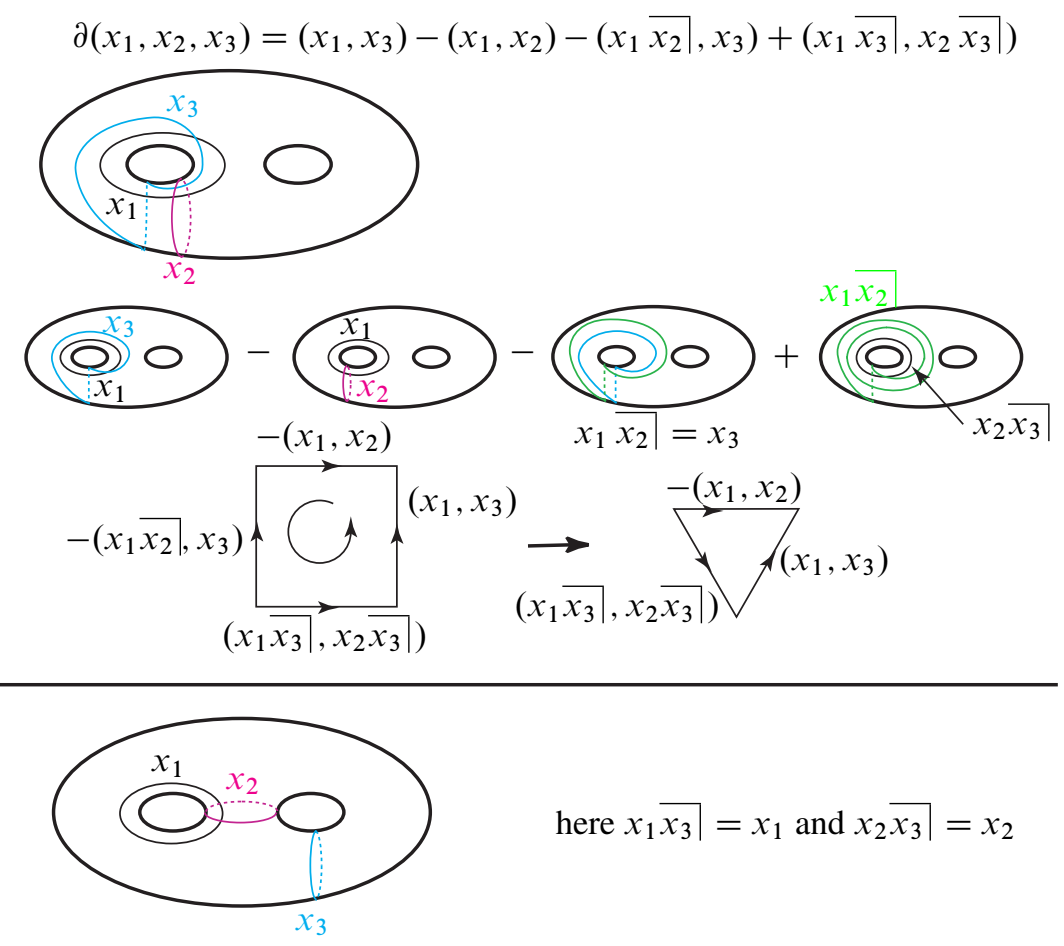

$$
\text { here } x_{1} \overline{x_{3}}=x_{1} \text { and } x_{2} \overline{x_{3}}=x_{2}
$$

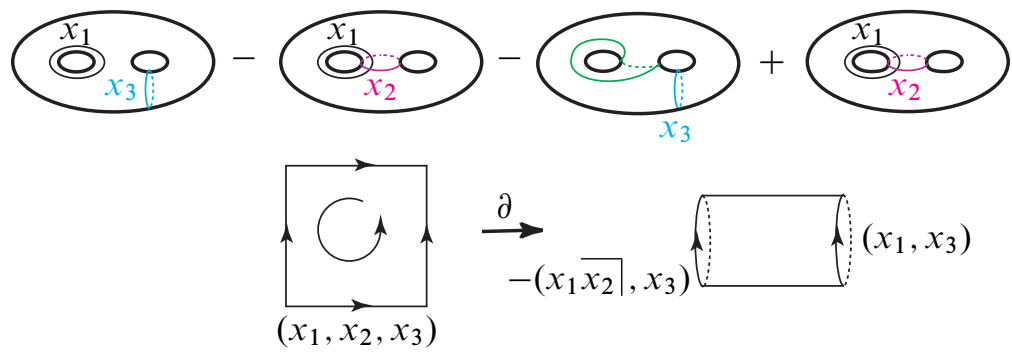

Figure 3: Boundaries of 3-simplices with identifications 


\subsection{Structures on the chain (sub)groups}

For the quandle chain complex we have some additional initial structures on the component groups $C_{n}(Q), Z_{n}(Q), B_{n}(Q)$, and $H_{n}(Q)$. Let $F$ be a fixed surface of genus $g$. Let $G=\operatorname{MCG}(F)$. Again, take $Q$ to be the Dehn quandle and let $\mathbb{Z}(G)$ be the integral group-ring of $G$. $G$ acts by homeomorphism on $F$ and on circles in $F$, and we define an action on $n$-tuples of circles representing $n$-simplices of $C_{n}$.

In keeping with the notation developed for individual Dehn twists, we write an arbitrary homeomorphism on the right of whatever object it is acting upon. For a homeomorphism $\alpha \in G$, and $\left(x_{0}, x_{1}, \ldots, x_{n-1}\right) \in C_{n}$, define $\left(x_{0}, x_{1}, \ldots, x_{n-1}\right) \alpha=$ $\left(\left(x_{1}\right) \alpha,\left(x_{2}\right) \alpha, \ldots,\left(x_{n-1}\right) \alpha\right)$. We extend this action linearly to $n$-chains, and linearly in $\mathbb{Z}(G)$. Since the action is induced from homeomorphisms, it preserves cycles, boundaries and homology classes. Then the groups $C_{n}(Q), Z_{n}(Q), B_{n}(Q)$, and $H_{n}(Q)$ are (right) $\mathbb{Z}(G)$-modules. Analogous statements can be made for the component groups of the rack and degeneracy complexes.

Since any homeomorphism $\alpha \in G$ is a composition of Dehn twists, by repeated use of quandle axiom (Q3) which governs the distributivity of Dehn twists over one another, one can check that the $\mathbb{Z}(G)$ action defined above actually commutes with the boundary operator. Thus, we may think of elements of $\mathbb{Z}(G)$ as giving chain maps $C_{*}(Q) \rightarrow C_{*}(Q)$, and also inducing maps on homology. In particular, single Dehn twists viewed as elements of $\mathbb{Z}(G)$ with coefficient 1 give isomorphisms on the chain and homology levels. We will make further use of this interpretation when we look at certain "stabilization" homomorphisms later.

\subsection{Cycles and homology in dimension 1}

Chains, cycles, and homology from $C_{1}(Q)$ are relatively simple to describe, but suggest aspects that may be useful in analyzing the higher dimensional cases. Some basic topological distinctions do show up in the chain $C_{1}(Q)$ and homology $H_{1}(Q)$ modules, reflecting transitivity properties of the action of $G=\operatorname{MCG}(F)$ on circles in $F$.

For the Dehn quandle of a general closed surface $F$ of genus $g$, the chain group $C_{0}(Q)=0 . C_{1}(Q)$ is generated by isotopy classes of single (nontrivial) circles in $F$. Thus for all $a \in C_{1}, \partial a=0$, so all 1-chains are cycles, in particular the 1-chain represented by a single isotopically nontrivial circle is a 1-cycle.

Theorem 2.1 Let $F$ be a closed orientable surface of genus $g$. Let $C_{i} \equiv C_{i}(Q ; \mathbb{Z})$ and $H_{i} \equiv H_{i}(Q ; \mathbb{Z})$, the quandle chain and homology groups for the Dehn twist quandle $Q$. 
(1) Any 1-chain of odd length represents a homology class in $H_{1}$. In particular a single nontrivial 1-simplex (that is, a single isotopically nontrivial circle) represents a 1-homology class.

(2) Any 1-chain with an unequal number of positive and negative terms represents a homology class in $H_{1}$.

(3) The collection of nonseparating circles in $F$ forms a cyclic $\mathbb{Z}(G)$-submodule of $C_{1}$ and also of $H_{1}$, generated by a single nonseparating circle.

(4) For pairs of integers $r, s$ with $r+s=g$, the collection of all separating circles bounding subsurfaces of genera $r$ and $s$ in $F$ forms a cyclic $\mathbb{Z}(G)$-submodule in both $C_{1}$ and $H_{1}$, generated by a single such circle.

(5) There is a decomposition for chains:

$$
C_{1}(Q)=A_{\text {nonsep }} \oplus \bigoplus_{r+s=g} A_{\text {sep }}(r, s)
$$

of the modules $C_{1}(Q)$ and a similar one for $H_{1}(Q)$ corresponding to equivalence classes of circles differing by homeomorphism, and to decomposition of the surface into pairs of homeomorphically equivalent subsurfaces.

Proof We saw above that any $1-$ chain is a $1-$ cycle. Let $c_{2} \in C_{2}$ be a $2-$ chain. We may assume any simplex in $c_{2}$ with coefficient $n>1$ has been written as a sum of $n$ simplices with coefficient 1 . So $c_{2}=\left(a_{1}, b_{1}\right) \pm\left(a_{2}, b_{2}\right) \pm \ldots \pm\left(\left(a_{r}, b_{r}\right)\right.$. For a single 2-simplex, $\left(a_{i}, b_{i}\right)$ assuming neither $a_{i}$ nor $b_{i}$ is isotopically trivial notice that $\partial\left(a_{i}, b_{i}\right)=a_{i}-a_{i} \overline{b_{i}} \mid=0$ if and only if $a_{i}=a_{i} \overline{b_{i}} \mid$, if and only if $\left|a_{i} \cap b_{i}\right|=0$. Otherwise $\partial\left(a_{i}, b_{i}\right)=a_{i}-a_{i} \overline{b_{i}}$ consists of a positive/negative pair of terms, since neither $a_{i}$ nor $a_{i} \overline{b_{i}}$ can be 0 . We proceed by induction on the length (number of terms) of the 2-chain $c_{2}$. Assume for an arbitrary such 2-chain $c_{2}$ of length $\leq r$, with $\partial c_{2} \neq 0$, that the boundary consists of $k \geq 1$ such positive/negative pairs of terms. Since any cancellation involves cancelling one positive and one negative term, $\partial c_{2} \neq 0$ must have an even number of terms. Suppose we adjoin a single new 2-simplex to get $c_{2}^{\prime}=c_{2} \pm\left(a_{n+1}, b_{n+1}\right)$ with $\partial c_{2}^{\prime} \neq 0$. Again, any cancellations will occur among positive/negative pairs, so by the inductive assumption we see $\partial c_{2}^{\prime}$ must have its remaining terms occurring in pairs. This proves (1) and (2). In particular, a single nontrivial circle represents a 1 -homology class.

For statements (3), (4), and (5), notice that $G$ acts transitively on all non-separating circles in $F$. Also for a given pair $r, s \in \mathbb{Z}$ with $r+s=g, G$ acts transitively on separating circles which bound subsurfaces of genera $r$ and $s$ in $F$, and the action preserves homology classes. The set of chains of non-separating circles (resp. chains of separating circles separating appropriate genus subsurfaces) are each closed under 
the $\mathbb{Z}(G)$ action. So single circles of these respective types generate disjoint cyclic $\mathbb{Z}(G)$-submodules, in both $C_{1}$ and in $H_{1}$.

\subsection{Cycles in dimensions $\geq 2$}

In dimensions $\geq 2$, the geometric interpretations and the algebra of cycles become more complex and more robust. Obviously, any boundary of an $n$-chain is an $(n-1)$ cycle. Somewhat more surprisingly we have the following:

Observation 2.2 Any single $n$-simplex $\sigma_{n}=\left(x_{1}, \ldots, x_{n}\right)$ for which all the entries (circles in $F$ ) are mutually disjoint, is a cycle. $\partial \sigma_{n}=0$ because for each entry $x_{2}, \ldots, x_{n}$, a twist about such a circle disjoint from all the others has no effect on the others, and so the terms arising in the second sum of the expression for the boundary come with opposite signs, and cancel those in the first sum. This generalizes the $n=1$ case and the generalization will be placed on a more formal footing in the later discussion of stabilization in Section 4.1. We will refer to such a simplex as a disjoint simplex.

We will now examine certain types of 2-cycles which seem to have a lot of structure. Specifically, we consider linear combinations of ordered pairs of the form

$$
z=\left(x_{1}, y_{1}\right) \pm\left(x_{2}, y_{2}\right) \pm \cdots \pm\left(x_{r}, y_{r}\right) .
$$

Here, a simplex with coefficient $n \neq \pm 1$ is written as a sum of $n$ simplices with coefficients \pm 1 . If all coefficients are positive, $x_{i} \overline{y_{i}}=x_{i+1}$, for $1 \leq i \leq r-1$, and $x_{r} \overline{y_{r}}=x_{1}$. Thus, the image of the first term in a pair, under the twist by the second term in the pair, is the first term of the next pair, with this process applying cyclically to the terms of the pairs in the linear combination. As described in Section 2.1, negative coefficients correspond to left twists about the second entry of a 2-simplex.

So a cycle $z=\left(x_{1}, y_{1}\right)+\left(x_{2}, y_{2}\right)+\cdots+\left(x_{r}, y_{r}\right)$ corresponds to a factorization of a homeomorphism $\phi \in G$, as a composition of right Dehn twists $\phi=\overline{y_{1}}\left|\overline{y_{2}}\right| \ldots \overline{y_{r}} \mid$, having the property that $\left(x_{1}\right) \phi=x_{1}$.

Generally, such cycles correspond to factorizations of reducible homeomorphisms of the surface $F$, and the circle $x_{1}$ is a component of the 1-dimensional submanifold of $F$ which is fixed by $\phi . x_{1}$ is a "test circle" and the intermediate $x_{i}$ 's form an abstract (discrete) geometric cycle, recording it's images under successive Dehn twists $\overline{y_{i}}$, or $\sqrt{y_{i}}$ in the factorization of $\phi$. For an example, see Figure 4 below. Any factorization of a homeomorphism $\phi$ fixing a circle $a$ yields a relation and a 2-cycle starting at $a$. In particular, it is easy to check the following: 
Proposition 2.3 The relations (F0), (F2), and (F3) yield 2-cycles, where $|a \cap b|=0$ and $\left|a_{i} \cap c\right|=1$ for all $i=1,2,3$, and $\left|a_{i} \cap a_{j}\right|=0$, for all $i, j$ :

(1) $(\mathrm{F} 0) \Leftrightarrow a \bar{b}|\bar{b}|=a \Leftrightarrow(a, b)+(a \bar{b} \mid, b)$

(2) $(\mathrm{F} 2) \Leftrightarrow c \overline{a_{1}}\left|\overline{a_{2}}, \bar{c}\right| \overline{a_{1}} \mid \overline{a_{2}}=c$

$\Leftrightarrow\left(c, a_{1}\right)+\left(c \overline{a_{1}}, a_{2}\right)$

$$
+\left(c \overline{a_{1}} \mid \overline{a_{2}}, c\right)+\left(c \overline{a_{1}}\left|\overline{a_{2}}\right| c \mid, a_{1}\right)+\left(c \overline{a_{1}}\left|\overline{a_{2}}\right| \bar{c} \overline{a_{1}}, a_{2}\right)
$$

$$
\begin{aligned}
& (\mathrm{F} 3) \Leftrightarrow c \overline{a_{1}}\left|\overline{a_{2}}\right| \overline{a_{3}}|\bar{c}| \overline{a_{1}}\left|\overline{a_{2}}\right| \overline{a_{3}}|\bar{c}| \overline{a_{1}}\left|\overline{a_{2}}\right| \overline{a_{3}}=c \\
& \Leftrightarrow\left(c, a_{1}\right)+\left(c \overline{a_{1}}, a_{2}\right)+\left(c \overline{a_{1}}\left|\overline{a_{2}}\right|, a_{3}\right)+\left(c \overline{a_{1}}\left|\overline{a_{2}}\right| \overline{a_{3}}, c\right) \\
& +\left(c \overline{a_{1}}\left|\overline{a_{2}}\right| \overline{a_{3}}|c|, a_{1}\right)+\left(c \overline{a_{1}}\left|\overline{a_{2}}\right| \overline{a_{3}}|\bar{c}| \overline{a_{1}} \mid, a_{2}\right) \\
& +\left(c \overline{a_{1}}\left|\overline{a_{2}}\right| \overline{a_{3}}|\bar{c}| \overline{a_{1}} \mid \overline{a_{2}}, a_{3}\right)+\left(c \overline{a_{1}}\left|\overline{a_{2}}\right| \overline{a_{3}}|\bar{c}| \overline{a_{1}}\left|\overline{a_{2}}\right| \overline{a_{3}}, c\right) \\
& +\left(c \overline{a_{1}}\left|\overline{a_{2}}\right| \overline{a_{3}} \vec{c}\left|\overline{a_{1}}\right| \overline{a_{2}}\left|\overline{a_{3}}\right| \bar{c}, a_{1}\right)+\left(c \overline{a_{1}}\left|\overline{a_{2}}\right| \overline{a_{3}}|\bar{c}| \overline{a_{1}}\left|\overline{a_{2}}\right| \overline{a_{3}}|\bar{c}| \overline{a_{1}} \mid, a_{2}\right) \\
& +\left(c \overline{a_{1}}\left|\overline{a_{2}}\right| \overline{a_{3}} \bar{c} \overline{a_{1}}\left|\overline{a_{2}}\right| \overline{a_{3}} \bar{c} \overline{a_{1}}\left|\overline{a_{2}}\right|, a_{3}\right)
\end{aligned}
$$

Figure 5 shows an example of relation (F3) and shows the particular symmetry inherent in it. Note that the intersection conditions for relations (F2) and (F3) require mutually disjoint circles, $a_{i}$. These can be disjoint copies of the same circle. As a consequence of the discussion above, we also have:

Proposition 2.4 Any group relation in the mapping class group $\underline{\operatorname{MCG}(F)}$, given by a series of Dehn twists, corresponds to a "universal" 2-cycle in $Z_{2}(Q)$.

Proof Write such a relation as a product of Dehn twists about circles, set equal to $1 \in \operatorname{MCG}(F)$. Since the relation is true in $\operatorname{MCG}(F)$, the product fixes all circles. From above, we may use any circle as the first entry of the first simplex. The first entries of successive simplices are its images under the twists. In the sense that the resulting $2-$ cycle can be built for any initial circle, it is universal.

One can represent 2-cycles via generalized knot diagrams, generalized in the sense that the diagrams may have strands they may not close up. Carter, Kamada and Saito [1] have a more sophisticated approach which allows them also to represent cycles of higher dimension using quandle colored knotted surfaces and regions. They show that for knot diagrams corresponding to 2-cycles, the diagrams represent homologous cycles iff the diagrams differ by colored variants of Reidemeister moves or appropriate cobordisms of such diagrams. Figure 6 gives the general naïve rubric under which this correspondence holds. See [1] for a more complete treatment. 

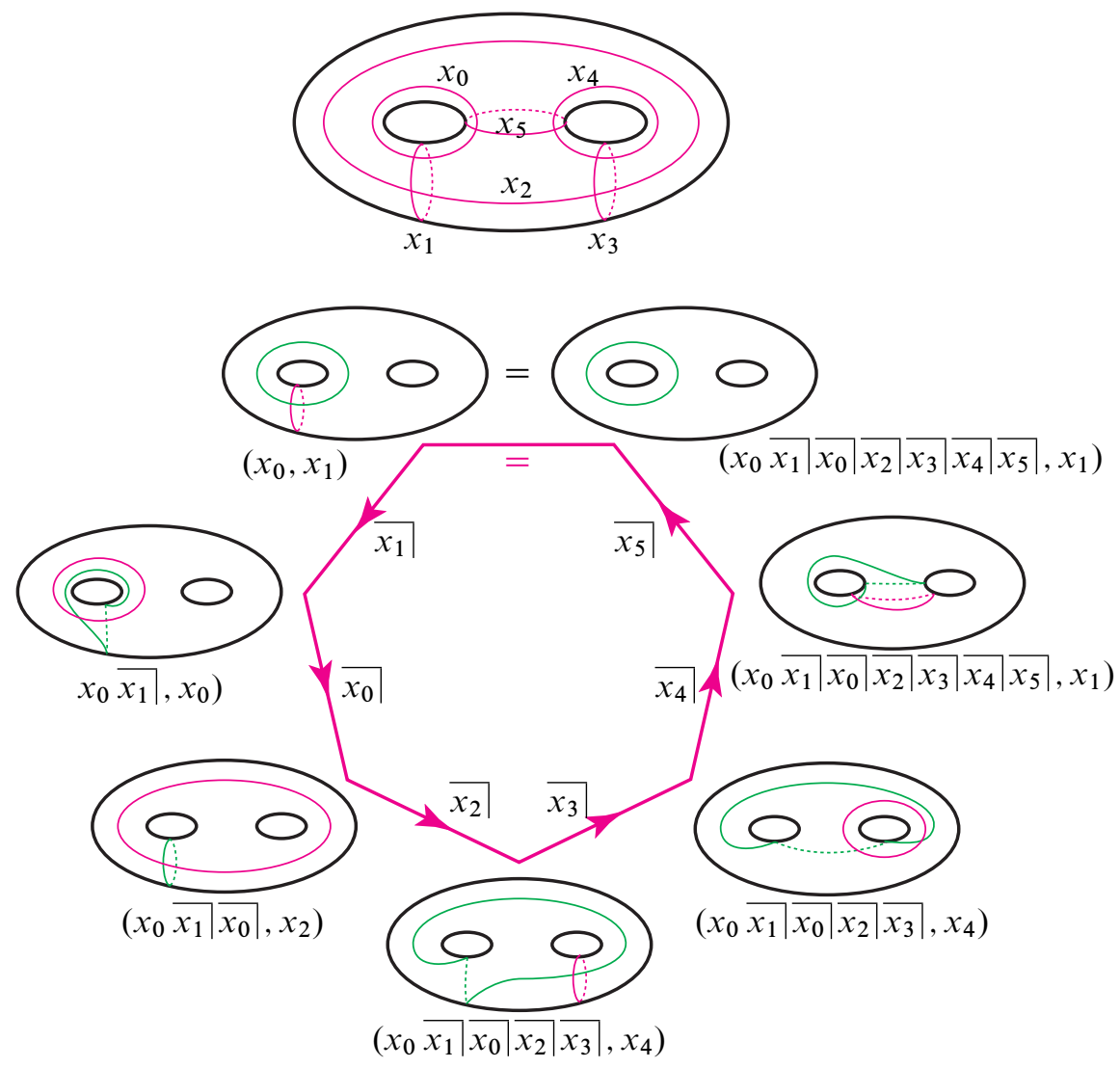

The homeomorphism $\phi=\overline{x_{1}}\left|\overline{x_{0}}\right| \overline{x_{2}}\left|\overline{x_{3}}\right| \overline{x_{4}} \mid \overline{x_{5}}$ fixing $x_{0}$ corresponds to the cycle

$$
\begin{aligned}
z=\left(x_{0}, x_{1}\right)+\left(x_{0} \overline{x_{1}}, x_{0}\right) & +\left(x_{0} \overline{x_{1}} \mid \overline{x_{0}}, x_{2}\right)+\left(x_{0} \overline{x_{1}} \overline{x_{0}}\left|\overline{x_{2}}\right|, x_{3}\right) \\
& +\left(x_{0} \overline{x_{1}}\left|\overline{x_{0}}\right| \overline{x_{2}} \mid \overline{x_{3}}, x_{4}\right)+\left(x_{0} \overline{x_{1}}\left|\overline{x_{0}}\right| \overline{x_{2}}\left|\overline{x_{3}}\right| \overline{x_{4}}, x_{5}\right)
\end{aligned}
$$

Figure 4: 2-cycle

Figure 7 shows the generalized knot diagram corresponding to the cycle of the relation (F3). Moves corresponding to the initial steps of the derivation in the proof of Theorem 1.8 are shown. The derivation began with moves modeled on the simpler diagram and moves associated to relation (F2) and Theorem 1.7.

It seems possible that these generalized diagrams might be realized as "virtual knot diagrams" (see Kauffman [6]) and actually become closed diagrams when viewed as diagrams on a surface of genus $\geq 1$, instead of projected on the plane or onto $S^{2}$. 
Cycle

$$
c \overline{a_{1}}\left|\overline{a_{2}}\right| \overline{a_{3}}\left|\bar{c} \overline{a_{1}}\right| \overline{a_{2}}\left|\overline{a_{3}}\right| \bar{c} \overline{a_{1}}\left|\overline{a_{2}}\right| \overline{a_{3}}=c
$$
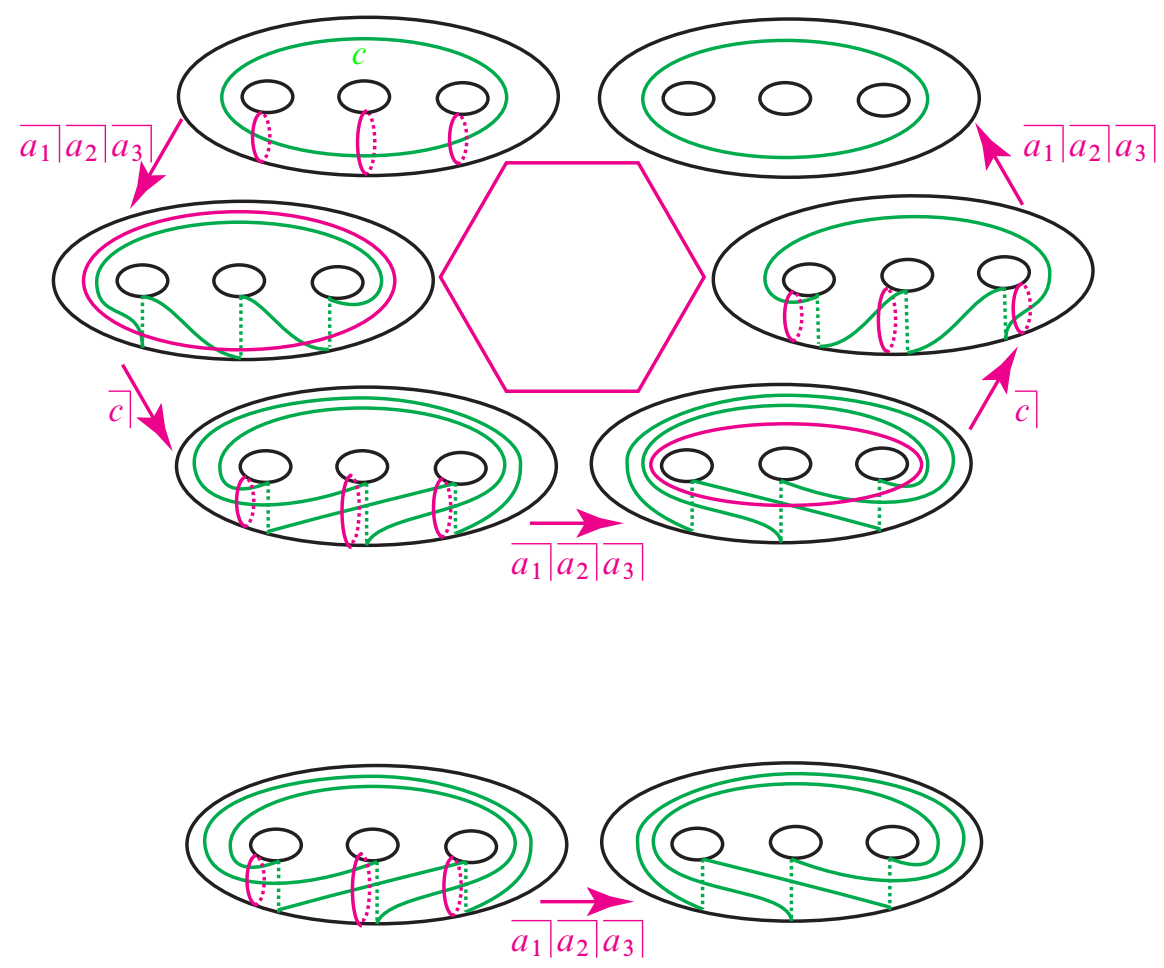

Symmetry

Figure 5: 2-cycle from relation (F3), and symmetry

This suggests the following:

Questions (1) Which quandle 2-cycle diagrams correspond to closed knot diagrams (that is, to diagrams that can be realized in $\mathbb{R}^{2}$ or $S^{2}$ )?

(2) For those that cannot be realized by closed diagrams in $\mathbb{R}^{2}$ or $S^{2}$, what does the minimal genus of the surface necessary to realize the diagram tell us about the 2-cycle? Is it some kind of invariant? 

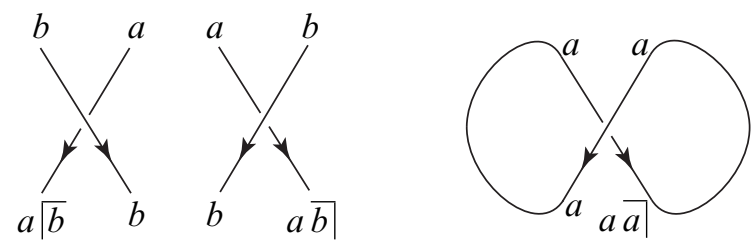

cycle: $(a, a)(=0)$

For $|a \cap b|=0$

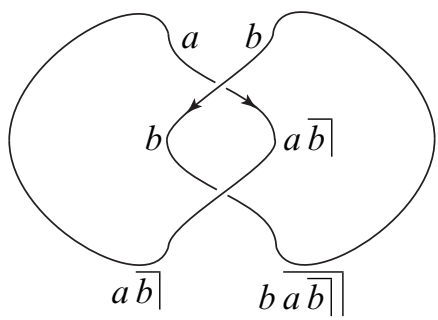

cycle: $(a, b)+(b, a \bar{b})$

since $b \bar{a} \bar{b} \mid=b$ and $a \bar{b}=a$

For $\left|x_{1} \cap x_{2}\right|=1$

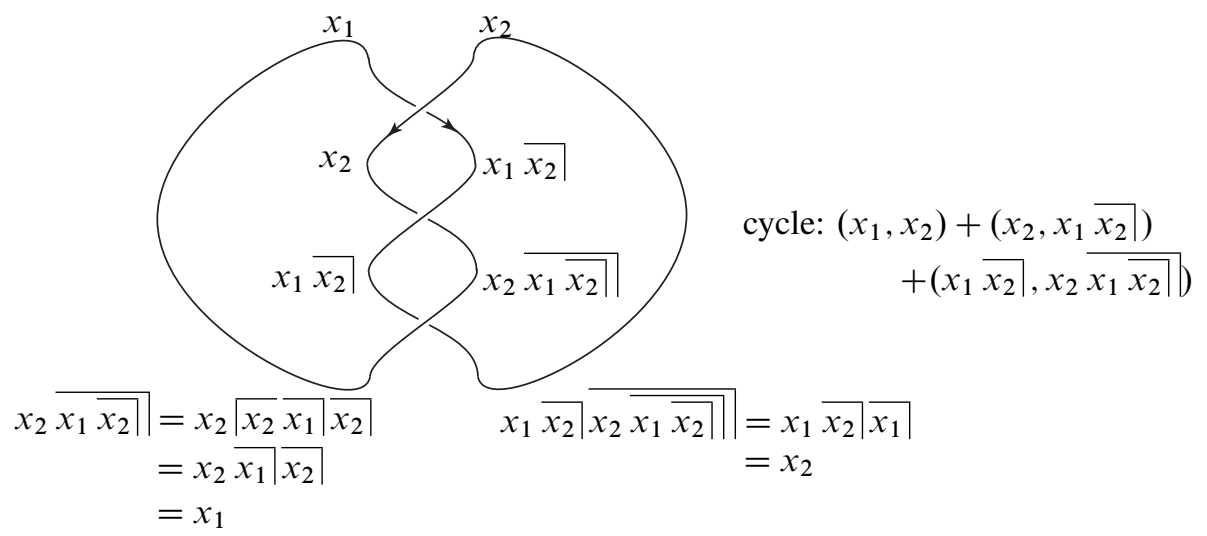

Figure 6: Cycles and knot diagrams

\section{Cycles, homology and module structure in dimensions $\geq 2$}

\subsection{Transitivity properties}

We now look at some further transitivity properties of the action of $\mathrm{MCG}(F)$ on circles. These will be used in the next section. Lickorish in [8] gives the following characterizations of the transitivity of $\operatorname{MCG}(F)$ on non-separating circles on the surface $F$. 

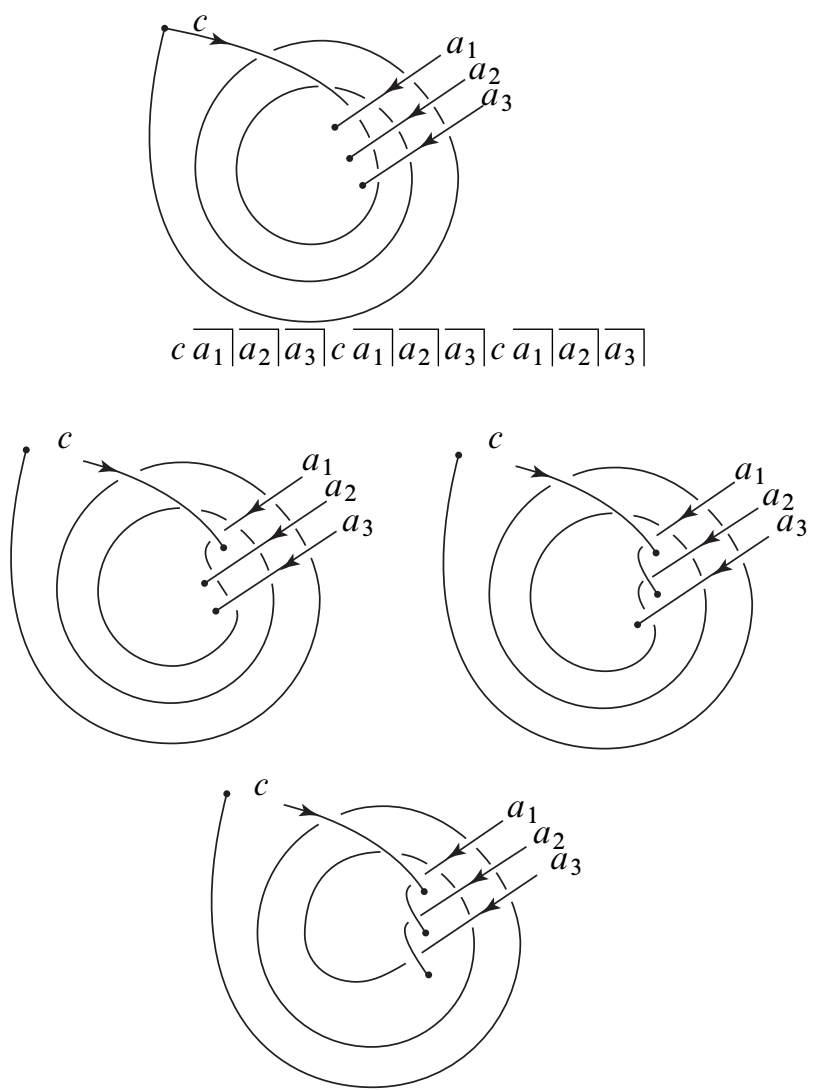

Initial moves for diagrams in the proof of the relation

$$
c \overline{a_{1}}\left|\overline{a_{2}}\right| \overline{a_{3}}\left|c \overline{a_{1}}\right| \overline{a_{2}}\left|\overline{a_{3}}\right| c \overline{a_{1}}\left|\overline{a_{2}}\right| \overline{a_{3}}=c
$$

Figure 7: Generalized diagram for relation (F3) and initial moves

Proposition 3.1 (Lickorish [8]) Suppose oriented, non-separating, simple closed curves $p$ and $q$ are contained in the interior of a surface $F$. Then there exists a homeomorphism $h \in \operatorname{MCG}(F)$, such that $(p) h=q$.

Corollary 3.2 (Lickorish [8]) Let $p_{1}, p_{2}, \ldots, p_{n}$ be disjoint simple closed curves in the interior of $F$, the union of which does not separate $F$. Let $q_{1}, q_{2}, \ldots, q_{n}$ be another set of curves with the same properties. Then there is a homeomorphism $h$ of $F$ that is in the group generated by twists, so that $\left(p_{i}\right) h=q_{i}$ for each $i=1,2, \ldots, n$.

Building on this we look at transitivity of $\operatorname{MCG}(F)$ on certain other simple configurations of circles in $F$. 

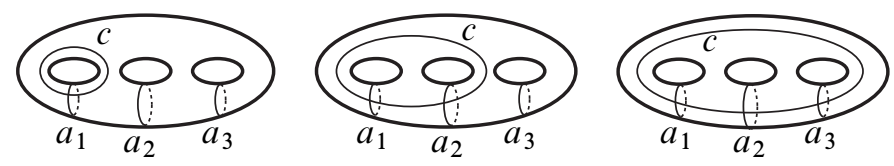

(0)

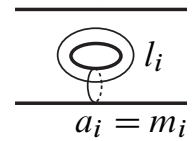

(1)

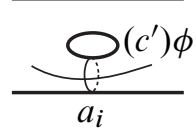

(2)

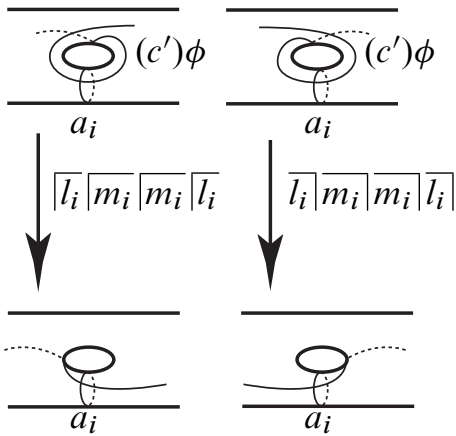

Figure 8: Standard position for tuples $\left(c ; a_{1}, \ldots, a_{t}\right)$ on surface. Genus 3 example is shown.

Proposition 3.3 Let $c, a_{1}, a_{2}, \ldots, a_{t}$ be circles in the interior of the surface $F$ of genus $g$, with $t \leq g$, such that $\left|c \cap a_{i}\right|=1$, for all $i$ with $1 \leq i \leq t$. Also assume $\left|a_{i} \cap a_{j}\right|=0$ and $a_{i} \neq a_{j}$ for all $i \neq j$ with $1 \leq i, j, \leq t$. Then $\operatorname{MCG}(F)$ acts transitively on tuples $\left(c ; a_{1}, \ldots, a_{t}\right)$.

Proof For each surface of genus $g$, let the curves $m_{i}$ denote the standard meridians, and $l_{i}$ be the standard longitudes. For each $t \leq g$, fix a standard tuple $\left(c ; a_{1}, \ldots, a_{t}\right)$ where the $a_{i}$ are standard successive meridians (so $a_{i}=m_{i}$ ) and $c$, are as shown in Figure 8 below, in the instance of a genus 3 surface. We will also say that an image of a curve $c^{\prime}$ is standard locally if it meets the $a_{i}$ 's as $c$ does, and the image is standard if the picture globally looks like one of the diagrams in the first row below. Suppose we have a tuple $\left(c^{\prime} ; a_{1}^{\prime}, \ldots, a_{t}^{\prime}\right) \subset F$. Corollary 3.2 gives us a homeomorphism $\phi$ such that for each $t$ with $1 \leq t \leq g$, we have $\left(a_{i}^{\prime}\right) \phi=a_{i}$. Since $\phi$ is a homeomorphism, it preserves intersections, so $\left|\left(c^{\prime}\right) \phi \cap a_{i}\right|=1$, for all $i=1 \ldots, t$. So a small neighborhood of $\left(c^{\prime}\right) \phi \cap a_{i}$ looks like of one of the regions shown in Figure 8, diagrams (1), (2), or (3). We have the following cases.

(1) As seen in diagram (1) of Figure $8,\left(c^{\prime}\right) \phi$ is already standard with respect to $a_{i}$.

(2) As seen in diagram (2) of Figure 8, applying the Dehn twists $\overline{l_{i}} \sqrt{m_{i}} \sqrt{m_{i}} \overline{l_{i}}$ fixes $a_{i}$ as a set, and yields the image of $\left(c^{\prime}\right) \phi$ shown below. These twists also have no effect on any other $a_{j}$. 
(3) As seen in diagram (3) of Figure 8, applying the Dehn twists $\sqrt{l_{i}} \sqrt{m_{i}} \sqrt{m_{i}} \sqrt{l_{i}}$ fixes $a_{i}$ as a set, and yields the image of $\left(c^{\prime}\right) \phi$ shown below. Again the twists have no effect on other $a_{j} \mathrm{~s}$.

We may then modify the remainder of the resulting image of $\left(c^{\prime}\right) \phi$ for the other $a_{j}$ similarly, getting the image of $c^{\prime}$ to locally be in standard position with respect to all the $a_{i}$. Then twists in the complement of $\left\{a_{1}, \ldots, a_{t}\right\}$ get the image of $c^{\prime}$ to globally coincide with $c$. Thus any tuple $\left(c^{\prime} ; a_{1}^{\prime}, \ldots, a_{t}^{\prime}\right)$ may be mapped to the standard tuple $\left(c ; a_{1}, \ldots, a_{t}\right)$, so the action of $\operatorname{MCG}(F)$ on such tuples is transitive.

\subsection{Module decompositions for cycles and homology}

In analogy to the situation which arose in the one dimensional cycle and homology modules, the transitivity of the $\mathrm{MCG}(F)$ action on various configurations of circles in $F$ allows us to peel off cyclic submodules in the cycle and homology modules in higher dimensions, corresponding to these configurations.

Theorem 3.4 (1) For each $n \in \mathbb{Z}$, there exists a cyclic $\mathbb{Z}(G)$ submodule, $C_{n}(0)$ of the cycle module $Z_{n}(Q)$, which corresponds to a disjoint $n$-simplex $\left(x_{1}, \ldots, x_{n}\right)$, where each $x_{i}$ is non-separating in $F$. It contains all other such disjoint $n$-cycles.

(2) The cycles of the types found in relations (F2) and (F3) yield submodules $C_{2}(2)$ and $C_{2}(3)$ respectively, of $Z_{2}(Q)$. The submodules are cyclic, generated respectively by tuples of the forms $\left(c ; a_{1}, a_{2}\right)$ and $\left(c ; a_{1}, a_{2}, a_{3}\right)$, corresponding to the relations. This yields a decomposition of the cycle module in dimension 2 as

$$
Z_{2}(Q)=C_{2}(0) \oplus C_{2}(1) \oplus C_{2}(2) \oplus C_{2}(3) \oplus M .
$$

In the the notation for the cyclic summands $C_{j}(k)$, the index $k$ is meant to recall the number of circles $a_{i}$ which intersect the circle $c$ once, in the given relation.

Proof (1) Following Observation 2.2, any such $n$-tuple represents a cycle in $Z_{n}(Q)$. By Lickorish's Corollary 3.2 above, for all $n \in \mathbb{Z}, G=\operatorname{MCG}(F)$ acts transitively on $n-$ tuples of mutually disjoint non-separating circles, and thus they are all equivalent under the $\mathbb{Z}(G)$ action on $Z_{n}(Q)$. So one such $n$-tuple generates a submodule containing all others.

(2) In a similar fashion, Proposition 3.3 says that $\operatorname{MCG}(F)$ acts transitively on the configurations of circles corresponding to the relations (F0), (F2), and (F3), and thus such configurations are equivalent under the $\mathbb{Z}(G)$ action as well. Any such configuration generates a submodule containing the others of its type, under the action, and the configurations of relations (F0), (F2), and (F3) are distinct, so the submodules intersect trivially. 
Even though the relations of this type do not seem continue past (F3), it seems conceivable that if there exist other relations associated to other configurations of circles, on which $\operatorname{MCG}(F)$ acted transitively, they would continue to yield cyclic submodules in the same fashion. From these considerations it seems reasonable to ask

Questions (3) Does $Z_{2}(Q)$ decompose completely into a sum of cyclic submodules each corresponding to a configuration of circles yielding a relation? For instance is $Z_{2}(Q)$ semisimple as a $\mathbb{Z}(G)$ module?

(4) What do "relations" look like for dimensions $>2$, and if they exist, do all the $Z_{n}(Q)$ decompose into cyclic submodules corresponding to such higher relations?

(5) Do there exist relations corresponding to more complicated configurations of circles, (in the sense of higher intersection numbers occurring) on which $\operatorname{MCG}(F)$ acts transitively?

Part (1) of Theorem 3.4 does suggest, with the ability to peel off cyclic summands corresponding to the disjoint simplices, that to at least a limited degree, such a decomposition may occur in the higher dimensions.

We now come to some results giving a characterization of a large class of 2-cycles which represent homology classes in $H_{2}^{Q}(Q)$. In [1], Carter, Kamada and Saito, invoking a split short exact sequence

$$
0 \rightarrow C_{n}^{D}(X) \stackrel{i}{\rightarrow} C_{n}^{R}(X) \stackrel{j}{\rightarrow} C_{n}^{Q}(X) \rightarrow 0
$$

and the resulting long exact homology sequence

$$
\cdots \stackrel{\partial_{*}}{\rightarrow} H_{n}^{D}(X: G) \stackrel{i_{*}}{\rightarrow} H_{n}^{R}(X: G) \stackrel{j_{*}}{\rightarrow} H_{n}^{Q}(X: G) \stackrel{\partial_{*}}{\rightarrow} H_{n-1}^{D}(X: G) \rightarrow \cdots,
$$

from an earlier paper, prove the following two theorems:

Theorem 3.5 (Carter, Kamada and Saito [1]) Let $X$ be a quandle. The boundary homomorphism $\partial_{*} H_{3}^{Q}(X) \rightarrow H_{2}^{D}(X)$ in the long exact sequence of quandle homology is trivial.

Theorem 3.6 (Carter, Kamada and Saito [1]) Let $X$ be a quandle. The boundary homomorphism $\partial_{*} H_{4}^{Q}(X) \rightarrow H_{3}^{D}(X)$ in the long exact sequence of quandle homology is trivial.

We will use this to show the following: 
Theorem 3.7 Any quandle 2-cycle in $Z_{2}(Q)$, or quandle 3-cycle in $Z_{3}(Q)$ which involves only positive or only negative terms represents a homology class in the quandle homology $\mathrm{H}_{2}(Q)$ or $\mathrm{H}_{3}(Q)$ respectively.

As in Theorem 3.4, these yield cyclic submodules, now in homology. First we observe this is true for rack homology in any dimension.

Proposition 3.8 Any rack $n$-cycle in $Z_{n}^{R}(Q)$ which involves unequal numbers of positive and negative simplices represents a homology class in the rack homology $H_{n}^{R}(Q)$.

Proof We state the proof for rack 2-cycles. An analogous argument holds in dimension $n$. Thus, in the rack chain complex, since degenerate simplices are not declared to be 0 , every 3 -simplex $\left(x_{1}, x_{2}, x_{3}\right)$ has boundary consisting of four nonzero terms. (In dimension $n, \partial\left(x_{1}, \ldots, x_{n}\right)$ has $2 n-2$ nonzero terms)

$$
\partial\left(x_{1}, x_{2}, x_{3}\right)=\left(x_{1}, x_{3}\right)-\left(x_{1}, x_{2}\right)-\left(x_{1} x_{2} \overline{x_{2}}, x_{3}\right)+\left(x_{1} \overline{x_{3}}, x_{2} \overline{x_{3}}\right)
$$

These occur in positive/negative pairs as shown. Any cancellation occurring when the boundary of a 2 -chain is taken eliminates positive and negative simplices in pairs. Thus it is not possible for the chain resulting from taking the boundary to have an unequal number of positive and negative simplices. Hence all cycles of this type represent elements in homology.

In particular, the cycles corresponding to the relations (F1), (F2), and (F3), when considered as rack 2-cycles give a decomposition of $H_{2}^{R}(Q)$ as a direct sum, analogous to that described for the quandle cycle module in Theorem 3.4

Proof of Theorem 3.7 We prove the statement for dimension 2. The proof in dimension 3 is analogous. We take $X$ to be the Dehn quandle $Q$ of a specified surface $F$. By the first theorem above, of Carter, Kamada, and Saito, $\partial_{*}=0$, and the tail of the long exact homology sequence looks like

$$
\cdots \longrightarrow H_{3}^{Q}(X) \stackrel{0}{\longrightarrow} H_{2}^{D}(X) \stackrel{i_{*}}{\longrightarrow} H_{2}^{R}(X) \stackrel{j_{*}}{\longrightarrow} H_{2}^{Q}(X) \stackrel{\partial_{*}}{\longrightarrow} H_{1}^{D}(X) \longrightarrow \cdots
$$

but $H_{1}^{D}(X)=0$ since there do not exist any degenerate 1 -chains. Thus $H_{2}^{Q}(X) \cong$ $H_{2}^{R}(X) / H_{2}^{D}(X)$.

Now any nontrivial quandle $2-$ cycle is also a rack 2 -cycle. So taking $z \in Z_{2}^{Q}(X)$ with only positive or only negative terms, we can consider it as a rack 2-cycle in $Z_{2}^{R}(X)$. But by Proposition 3.8 above, all elements of $Z_{2}^{R}(Q)$ with unequal numbers 
of positive and negative terms represent homology classes in $H_{2}^{R}$. Since in the short exact sequence above, $j: C_{2}^{R}(X) \rightarrow C_{2}^{Q}(X)$ yields $C_{2}^{Q}(X) \cong C_{2}^{R}(X) / C_{2}^{D}(X)$, and since $C_{2}^{D}(X)$ consists only of chains of degenerate simplices, the image of a purely non-degenerate rack-chain such as $z$ under $j$ is the same nondegenerate chain viewed as a quandle-chain. Letting $[z]$ denote a homology class, on the level of homology, $j_{*}:[z]_{R} \mapsto[z]_{Q} \neq 0 \in H_{2}^{Q}(Z)$, since $[z]_{R}$ had no degenerate simplices to begin with. Thus such a $z$ as above represents a nonzero class $[z]_{Q} \in H_{2}^{Q}(X)$. A similar argument, invoking the second theorem of Carter, Kamada, and Saito mentioned above, gives the result in dimension 3.

Together with the results from Theorem 2.1, this gives the following:

Corollary 3.9 Individual disjoint simplices in dimensions 1,2, and 3 represent homology classes in those dimensions.

This will have further resonance with the "stabilization" results of the next section, in the presence of which, the obvious conjecture will arise.

\section{Further structure on the quandle chain complex}

\subsection{Stabilization, analogies and consequences}

Recall from the remarks in Section 2.2 that the action on $n$-simplices given by

$$
\left(x_{1}, \ldots, x_{n}\right) \bar{a}=\left(x_{1} \bar{a} \mid \ldots, x_{n} \bar{a}\right)
$$

which underlay the $\mathbb{Z}(G)$-module structure on the quandle chains and homology, commutes with the boundary operator, and can be considered as a chain map on $C_{*}(Q)$. We now introduce another family of homomorphisms on $C_{*}(Q)$ which are "compatible" with this action by Dehn twists and homeomorphisms. For a given element $a \in Q$, define maps $S_{n}^{\prime a}: C_{n}(Q) \rightarrow C_{n+1}(Q)$, on a single $n$-simplex, by $S_{n}^{\prime a}\left(x_{1}, \ldots, x_{n}\right)=\left(x_{1}, \ldots, x_{n}, a\right)$, and extend by linearity. Define $S_{n}^{a}=(-1)^{n+1} S_{n}^{\prime a}$. For reasons that will become more apparent from the results below, the maps $S_{n}^{a}$ will be called stabilizations.

Proposition 4.1 The family of $S_{n}^{a}$ 's give a chain homotopy between the identity on $C_{*}(Q)$ and the maps $\vec{a}$. 
Proof Consider an $n$-simplex $\left(x_{1}, \ldots, x_{n}\right) \in C_{n}(Q)$. Note that in the two portions of the sum which comprise the boundary of a simplex, the last term of the first part and the last term of the second part always have opposite signs. Then

$$
\begin{aligned}
\partial_{n+1} S_{n}^{a}\left(x_{1}, \ldots, x_{n}\right)= & \partial_{n+1}(-1)^{n+1}\left(x_{1}, \ldots, x_{n}, a\right) \\
= & (-1)^{n+1}\left[\left(x_{1}, x_{3}, \ldots, x_{n}, a\right)-\left(x_{1}, x_{2}, x_{4} \ldots, x_{n}, a\right)+\cdots\right. \\
& \pm\left(x_{1}, x_{2}, \ldots, x_{n-1}, a\right) \mp\left(x_{1}, x_{2}, \ldots, x_{n}\right) \\
& -\left(x_{1} \overline{x_{2}}, x_{3}, \ldots, x_{n}, a\right)+\left(x_{1} \overline{x_{3}}, x_{2} \overline{x_{3}}, x_{4}, \ldots, x_{n}, a\right)-\ldots \\
& \mp\left(x_{1} \overline{x_{n}}, x_{2} \overline{x_{n}}, \ldots, x_{n-1} \overline{x_{n}}, a\right) \\
& \left. \pm\left(x_{1} \bar{a}, x_{2} \bar{a}, \ldots, x_{n} \bar{a}\right)\right]
\end{aligned}
$$

while

$$
\begin{aligned}
S_{n-1}^{a} \partial_{n}\left(x_{1}, \ldots, x_{n}\right)= & S_{n-1}^{a}\left[\left(x_{1}, x_{3}, \ldots, x_{n}\right)-\left(x_{1}, x_{2}, x_{4}, \ldots, x_{n}\right)+\cdots\right. \\
& \pm\left(x_{1}, x_{2}, \ldots, x_{n-1}\right)-\left(x_{1} \overline{x_{2}}, x_{3}, \ldots, x_{n}\right) \\
& +\left(x_{1} \overline{x_{3}}, x_{2} \overline{x_{3}}, x_{4}, \ldots, x_{n}\right)-\ldots \\
& \left. \pm\left(x_{1} \overline{x_{n}}, x_{2} \overline{x_{n}}, \ldots, x_{n-1} \overline{x_{n}}\right)\right] \\
= & (-1)^{n}\left[\left(x_{1}, x_{3}, \ldots, x_{n}, a\right)-\left(x_{1}, x_{2}, x_{4} \ldots, x_{n}, a\right)+\cdots\right. \\
& \pm\left(x_{1}, x_{2}, \ldots, x_{n-1}, a\right)-\left(x_{1} \overline{x_{2}}, x_{3}, \ldots, x_{n}, a\right) \\
& +\left(x_{1} \overline{x_{3}}, x_{2} \overline{x_{3}}, x_{4}, \ldots, x_{n}, a\right)-\ldots \\
& \left.\mp\left(x_{1} \overline{x_{n}}, x_{2} \overline{x_{n}}, \ldots, x_{n-1} \overline{x_{n}}, a\right)\right]
\end{aligned}
$$

adding these expressions, only the underlined terms remain, so

$$
\partial_{n+1} S_{n}^{a}\left(x_{1}, \ldots, x_{n}\right)+S_{n-1}^{a} \partial_{n}\left(x_{1}, \ldots, x_{n}\right)=\left(x_{1}, \ldots, x_{n}\right)[ \pm(\operatorname{Id}-\bar{a})] .
$$

Thus, in particular we have an initial interpretation for homology of certain chains; If two $n$-cycles differ by a Dehn twist (or composition thereof) they are homologous. Then, by Corollary 3.9, all disjoint 2-simplices made of non-separating circles represent the same homology class in $H_{2}(Q)$, and similarly for non-separating circles when viewed individually as cycles in $H_{1}(Q)$. This suggests that similar behavior should occur among disjoint simplices in any dimension. We look now at some further evidence in this direction, involving the maps $S_{*}^{a}$, for a given $a \in Q$.

Recall that "circle" means isotopy class of a circle.

Lemma 4.2 For a given surface $F$, and fixed circle $a \in F$, let

$$
T_{a}=\{x \in Q \mid x \neq a, \text { and }|x \cap a|=0\} .
$$


(1) For each $a \in F, T_{a}$ is a sub-quandle of $Q$.

(2) For a given $a \in F$, the collection of chains in $C_{*}(Q)$ consisting of simplices each of whose entries is disjoint and distinct from $a$, forms a sub-complex $C_{*}^{a}(Q)$, with respect to $\partial$.

The associated cycle, boundary, and homology groups are denoted $Z_{*}^{a}(Q), B_{*}^{a}(Q)$, and $H_{*}^{a}(Q)$ respectively.

Proof (1) Fix a circle $a \in F$. Since the quandle operation and axioms are inherited from $Q$, we need to show $T_{a}$ is closed with respect to the operation. Suppose $b, c \in T_{a}$,

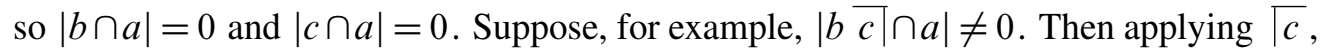
we'd have $|b \overrightarrow{c|| c} \cap a \sqrt{c}| \neq 0$, since the homeomorphism $\sqrt{c}$ preserves intersections. But then by $(\mathrm{Q} 2)$ and the fact that Dehn twists by non-intersecting circles have no effect, we'd have $|b \cap a| \neq 0$. Contradiction. Now suppose there exists a composition of Dehn twists about $c_{i}$ 's in $T_{a}$ (without loss of generality, assume they are right twists) $\overline{c_{1}} \ldots \overline{c_{n}}$ of minimal length, such that $b \overline{c_{1}} \mid \ldots \overline{c_{n}}=a$. By assumption, $\left|c_{n} \cap a\right|=0$, so $b \overline{c_{1}} \ldots \overline{c_{n-1}} \mid=a \overline{c_{n}}=a$. But then we have decreased the length of the supposedly minimal composition yielding $a$. Contradiction.

(2) The boundary operator involves only right Dehn twists. If the elements about which we twist and the ones on which they act, entries in the simplices of $C_{*}^{a}(Q)$, all belong to $T_{a}$, part 1 above insures that all the entries in the simplices which are images under $\partial$, will also lie in $T_{a}$, thus we have $\partial_{n}: C_{n}^{a}(Q) \rightarrow C_{n-1}^{a}(Q)$.

We can now show that $S_{n}^{a}$ promotes $n$-cycles disjoint from $a$ to $(n+1)$-cycles.

Proposition 4.3 For a given element $a \in Q$, and for all $n \geq 0$, the image $S_{n}^{a}\left(Z_{n}^{a}(Q)\right)$ lies in $Z_{n+1}(Q)$, and the map is injective.

Proof Recall that the quandle boundary operator has two pieces. The first piece looks like the standard simplicial homology boundary operator, which successively eliminates entries in the simplices and forms and alternating sum. The second piece brings in the quandle action, applying later elements to earlier ones, and again forms an alternating sum of the terms.

Acting on an $n$-cycle $z$ lying in $Z_{n}^{a}(Q), S_{n}^{a}$ adjoins the element $a$ in the $(n+1)$ st position of each term of $z$. Let $(z ; a)$ denote the image, $S_{n}^{a}(z)$. There are three types of terms in $\partial_{n+1}(z ; a)$.

(1) Terms of the form $\left(\partial_{n} z ; a\right)$ which come from the portions of the boundary operator action which don't involve the $(n+1)$ st term, $a$. 
(2) Terms of the form $(z ; \hat{a})$ coming from the first part of the boundary operator $\partial_{n+1}$ affecting the $(n+1)$ st element $a$.

(3) Terms of the form $\left(z \bar{a} \mid\right.$ ), coming from the second part of $\partial_{n+1}$ applying $\bar{a}$ to the previous terms.

Since all the entries in the terms of $z$ lie in $T_{a}$ and hence are disjoint and distinct from $a$, terms of types 2 and 3 cancel one another, due to the sign offset between the pieces of $\partial_{n+1}$, and the fact that in each term, $\bar{a} \mid$ acts trivially on all the other entries. Also, in all the terms, the entries prior to $a$ lie in $T_{a}$, and by Lemma 4.2 above, $T_{a}$ is closed under the quandle operations. Thus the entries arising from the later entries acting on the earlier ones, by the quandle operation, also lie in $T_{a}$. Since these were all entries in the terms of $z$, an $n$-cycle, their signs were such that they would have cancelled one another out as $\partial_{n}$ was applied. As it is, they still do, the only difference being that each such term now has an $a$ at the end of it. Nonetheless, due to the signs on the original terms, complete cancellation still occurs. By the definition, $S_{*}^{a}$ is injective, since none of the entries occurring before $a$ in any term in the image, are changed.

Gathering together these results and some from Section 3, we have the following:

Conjecture 4.4 (1) For all $n, n$-cycles involving only positive or only negative terms are homology representatives in $H_{n}(Q)$.

(2) In particular, for $n \geq 1$, a single disjoint $n$-simplex represents a homology class in $H_{n}(Q)$.

(3) For all $n$, If $z_{n} \in H_{n}(Q)$ is a homology representative and the circle $a$ is disjoint from all simplices of $z_{n}$, then the stabilization maps $S_{n}^{a}$, promote $z_{n}$ to an $(n+1)$-homology representative.

By Theorem 3.7 and Proposition 4.3 above, statements (1), (2), and (3) hold for $n \leq 3$. If statement (3) holds in general, these stabilization maps would behave somewhat like suspension isomorphisms. The ability to continue to stabilize "disjointly" would depend on the genus of $F$, the dimension of the initial chain, and the specific entries in the simplices.

Remark 4.5 One may think of a braid as describing the continuous motion, over time, of a set of $n$ objects (points), which are not allowed to intersect, in some space. If instead, we take the objects to be circles on the surface $F$, and the motion to be discrete, mediated by successive applications of Dehn twists, we have the Dehn quandle action. Pure braids are those sequences of twists of strands which return an ordered set of points to its original configuration. Quandle 2-cycles consist of sequences of 
successive Dehn twists which return a circle (or collection of circles) to its original position. So one may think of factorizations of a reducible homeomorphisms and the circles they fix, on which the 2-cycles are based, as corresponding to pure braids.

The quandle 2-cycles of the types mentioned here, specifically those associated to the relations (F0), (F2), and (F3), and the other examples shown, for example, in Figure 6, are all based on right hand Dehn twists. They would correspond to strictly positive pure braids. The positivity of the twists will be given added significance in the next section. It is not immediately clear how to interpret higher dimensional cycles in this context; perhaps as pure braids in higher dimensional braid groups.

The analogy to braids is strengthened by the existence of the stabilization maps $S_{n}^{a}$. In the chain complex $C_{*}^{a}$, of the chains disjoint from a given circle $a$, the analogy is almost exact. Braid stabilization adds a new un-entwined strand to the given braid. In $C_{*}^{a}$, we modify chains by adjoining a disjoint circle, which does not interact with the others. Generally though, in applications of $S_{n}^{a}$, the element $a$ is not necessarily disjoint from the other elements involved.

\section{A connection with Lefschetz fibrations of 4-manifolds}

\subsection{Background}

We give here the definitions and rudiments of the theory of Lefschetz fibrations. From Fuller [3] we have

Definition 5.1 A Lefschetz fibration on a smooth, compact, connected, oriented 4manifold $X$, over a smooth compact oriented 2-manifold $B$, is a map $\pi: X \rightarrow B$, such that at each critical point of $B$, there is an orientation-preserving chart on which $\pi: \mathbb{C}^{2} \rightarrow \mathbb{C}$ is given by $\pi(w, z)=w z$.

There are a finite number of critical values, say $\left\{b_{1}, \ldots, b_{n}\right\} \in B$. Preimages of regular values are fibers $F$, all diffeomorphic to a model surface $\Sigma_{g}$ of fixed genus $g . g$ is said to be the genus of the fibration. At the critical values, the singular fibers are immersed surfaces with a single transverse self-intersection, here assumed to be relatively minimal, that is, containing no imbedded sphere of self-intersection -1. In the discussions below, we will consider Lefschetz fibrations with $B=D^{2}$, the 2-disk, and $B=S^{2}$. The critical values all lie in the interior of $B$.

If we consider a disk $D^{2}$ in $B$ containing a single critical value $b_{i}$, the the preimage $\pi^{-1}\left(b_{i}\right)$ is a singular fiber of the Lefschetz fibration. It may be thought of as a copy 
of the fibering surface $F$, in which a specified simple closed curve (circle) $c_{i} \in F$ has been shrunk to a point. Each singular fiber has its own such specified curve, (and each may be distinct from the others), called the vanishing cycle of the fiber. As one approaches the singular fiber through regular fibers, the circles corresponding to the vanishing cycle may be thought of as shrinking to a point.

The Lefschetz fibration $\pi: X \rightarrow B$ can be thought of as being constructed by forming $B \times \Sigma_{g}$ and then attaching 2-handles along the curves corresponding to the vanishing cycles of the singular fibers, with appropriate framing. Following Fuller [3] or Gompf and Stipsicz [4], the topology around a singular fiber may be described as follows. Over a small disk neighborhood $D_{i}^{2}$ of the critical value $b_{i}$, we have a $\Sigma_{g}$ bundle, with a single singular fiber $F_{i}$, and a nonsingular surface bundle over the circle $S^{1}=\partial D_{i}^{2}$. This may be thought of as a mapping torus, where the bundle $\Sigma_{g} \times I$ is glued via the homeomorphism of $\Sigma_{g}$ determined by a right handed Dehn twist $\overline{c_{i}}$ about the circle $c_{i}$ representing the vanishing cycle of the singular fiber $F_{i}$. Thus for each singular fiber $F_{i}$ associated to the critical value $b_{i}$ we have the restriction

$$
\left.\pi^{-1}\right|_{\partial D_{i}^{2}}=\frac{\Sigma_{g} \times I}{(p, 0) \sim\left((p) \overline{\left.c_{i} \mid 1\right)}\right.} \quad p \in \Sigma_{g} .
$$

This prescription of the glueing for the surface bundle over $S^{1}=\partial D_{i}^{2}$ is the monodromy of the singular fiber, basically giving a homomorphism $\pi_{1}\left(B-\left\{b_{i}\right\}\right) \rightarrow \operatorname{MCG}\left(\Sigma_{g}\right)$.

If a Lefschetz fibration $\pi: X \rightarrow B$ has more than one singular fiber, it may be described as an appropriate gluing up of the fibered neighborhoods arising about the individual singular fibers, each with its specified vanishing cycle and associated local monodromy. If $b_{1}, \ldots, b_{n} \in B$ are the critical values, consider small mutually disjoint disks $D_{i} \subset B$ about the $b_{i}$ 's. Each has a Lefschetz fibration with exactly one singular fiber, and the associated vanishing cycle and monodromy about its boundary circle. If $b_{0} \in B$ is a regular value, we may take the critical values $b_{i}$ to be arranged in a circle about $b_{0}$, with the indices $i$ appearing in order as we traverse this circle counterclockwise. Let $\gamma_{i}$ be a collection of mutually disjoint arcs in $B$, joining the regular value $b_{0}$ to each $b_{i}$. Assuming a fixed identification of the the regular fiber $F_{0}=\pi^{-1}\left(b_{0}\right)$, with the model surface $\Sigma_{g}$, the trivial bundles above the $\gamma_{i}$ allow transport of this identification to regular fibers near each singular fiber. A regular neighborhood of the entire ensemble consisting of the regular point, $b_{0}$, the arcs $\gamma_{i}$, the disks $D_{i}$ (each containing its critical value $b_{i}$ ), is then a disk $D$ containing all the critical values. $\partial D$ deformation retracts to the product, in order, of the counterclockwise oriented loops which go around the critical points $b_{i}$, based at $b_{0}$. This then describes the global monodromy of the Lefschetz fibration, the image of the homomorphism $\pi_{1}\left(D-\left\{b_{1}, \ldots, b_{n}\right\}\right) \rightarrow \operatorname{MCG}\left(\Sigma_{g}\right)$. Traversing the boundary circle $\partial D$ once in the 
counterclockwise direction then corresponds to the element of $\operatorname{MCG}(F)$ given by the product (in order) of the individual Dehn twists around the vanishing cycles for the singular fibers, $\psi=\overline{c_{1}} \cdot \overline{c_{2}} \mid \cdots \overline{c_{n}}$. Thus the surface bundle over the boundary circle $\partial D$ may be described by

$$
\frac{\Sigma_{g} \times I}{(p, 0) \sim((p) \psi, 1)} \quad p \in \Sigma_{g},
$$

and the space $X$ can be described as

$$
\left(\Sigma_{g} \times B\right) \cup \bigcup_{i=1}^{n}(2 \text {-handles }) .
$$

The ordered list of vanishing cycles, twists about which give the global monodromy of a Lefschetz fibration, almost determines the fibration, but not quite. Such list may be permuted cyclically and still yield the same fibration. Also the choice of homeomorphism identifying the model surface $\Sigma_{g}$ with a typical regular fiber $F$ may be changed. This has the effect of conjugating each of the Dehn twists in the list corresponding to the monodromy, by a fixed element of $\operatorname{MCG}(F)$. Another possible modification of the initial data, which still yields the same fibration is given by applying elementary transformations. These have the effect of modifying the arcs $\gamma_{i}$ from the basepoint $b_{0}$ to each of the critical values $b_{i}$, where $i=1 \ldots n$. This modification is essentially sliding the $b_{0}$ endpoint of one such arc up along another adjacent one, and back down the other side, to the basepoint. If the $n$-tuple corresponding to the global monodromy is initially given by the $n$-tuple of vanishing cycles $\left[c_{1}, \ldots c_{i}, c_{i+1}, \ldots, c_{n}\right]$, performing such an elementary transformation yields the new $n$-tuple of vanishing cycles $\left[c_{1}, \ldots, c_{i+1}, c_{i} \overline{c_{i+1}}, \ldots, c_{n}\right]$. So the monodromy about individual singular fibers is changed, but the global monodromy in unaltered. Two Lefschetz fibrations are isomorphic iff the monodromy $n$-tuple of one can be obtained from the $n$-tuple of the other by a sequence of elementary transformations (and their inverses) and conjugation of all Dehn twists by a fixed element of $\operatorname{MCG}\left(\Sigma_{g}\right)$. See [4].

Citing theorems of Gompf and Donaldson, Fuller [3] and Gompf-Stipsicz [4] state the requirement that the monodromy homeomorphisms be given by products of right handed Dehn twists yields further structure on the Lefschetz fibered space $X$, for genus $g \geq 2$; it carries a symplectic structure, as well.

\subsection{Dehn quandle cycles, homology and Lefschetz fibrations}

Having given some basics relevant to Lefschetz fibrations, we now consider some initial connections to Dehn quandle chains and homology. Note that the requirement that the monodromy of a Lefschetz fibration be given by a product of purely right handed 
Dehn twists is in keeping with the the use of such twists in the description of the Dehn quandle boundary operator.

Following Fuller in [3], the topology of a neighborhood of an individual singular fiber of a Lefschetz fibration is completely determined by the associated vanishing cycle for the singular fiber. In particular the fiber neighborhoods determined by any two non-separating vanishing cycles are diffeomorphic, due to the transitivity of the action of $\operatorname{MCG}(F)$ on such non-separating curves; similarly also, for separating curves which separate $\Sigma_{g}$ in to pairs of sub-surfaces of the same genera. Thus:

Observation 5.2 For a specified surface $\Sigma_{g}$ with specified vanishing cycles, there is an exact correspondence between the diffeomorphism types of neighborhoods of singular fibers and the cyclic submodules in the decomposition of $H_{1}(Q)$, as described in Theorem 2.1 above.

We now come to the main result of this section. We show a correspondence between isomorphism classes of Lefschetz fibrations of genus $g$ with specified monodromy, and homology classes in $H_{2}(Q)$ where $Q$ is the Dehn quandle of the surface $\Sigma_{g}$. Let $\left[c_{1}, \ldots, c_{n}\right]$ denote the ordered sequence of circles, such that the product $\overline{c_{1}} \cdots \overline{c_{n}}$ represents the global monodromy of a genus $g$ Lefschetz fibration, $\pi: X \rightarrow B$. Choose a "test" circle $x \in \Sigma_{g}$ (whose successive images under the twists of the homeomorphism are the first terms in successive simplices of the chain). Consider the map to quandle 2-chains $\{$ monodromy homeomorphisms $\} \rightarrow C_{2}(Q)$, given by

$$
f_{x}:\left[c_{1}, \ldots, c_{n}\right] \mapsto\left(x, c_{1}\right)+\left(x \overline{c_{1}}, c_{2}\right)+\cdots+\left(x \overline{c_{1}} \cdots \overline{c_{n-1}}, c_{n}\right) .
$$

Here, the image of the the first entry in a given simplex, under right Dehn twisting by the second entry, gives the first entry in the next simplex. Choice of a test circle "anchors" the chain (cycle). We then have the following:

Theorem 5.3 Let $\Sigma_{g}$ be a fixed oriented surface of genus $g$, with circle $a \subset \Sigma_{g}$, and consider genus $g$ Lefschetz fibrations over the disk, $\pi: X \rightarrow D^{2}$.

(1) Suppose we have two monodromy $n$-tuples corresponding to a reducible homeomorphism $\psi$, with $(a) \psi=a$. If these $n$-tuples represent isomorphic Lefschetz fibrations with fiber $F \cong \Sigma_{g}$, their images under corresponding maps $f_{a}$ are homologous 2-cycles in $Z_{2}(Q)$, and represent the same homology class (generator) in $\mathrm{H}_{2}(Q)$.

(2) For a specified Lefschetz fibration monodromy $n$-tuple, and elements $a$ as in 1, there is a 1-1 correspondence between the images under $f_{a}$ and incompressible 
tori in the mapping torus

$$
\partial X=\frac{\Sigma_{g} \times I}{(p, 0) \sim((p) \psi, 1)} \quad p \in \Sigma_{g}
$$

Proof (1) Let $\left[c_{1}, \ldots, c_{n}\right]$ be an ordered $n$-tuple of circles on $\Sigma_{g}$ representing the global monodromy of a Lefschetz fibration over $D^{2}$. From Gompf and Stipsicz [4], cyclic permutations of such an $n$-tuple can be realized, up to conjugation by elementary transformations. Also by earlier remarks, any pair of monodromies representing isomorphic Lefschetz fibrations differ by elementary transformations and conjugation by elements of $\operatorname{MCG}\left(\Sigma_{g}\right)$. So we need to show that if two such $n$-tuples differ by elementary transformations and conjugation, then the corresponding images under appropriate maps, $f_{a}$, are homologous cycles in $Z_{2}(Q)$. We consider conjugation first.

Recall that by abuse of notation, each element $c_{i}$ in a monodromy $n$-tuple really represents a Dehn twist $\overline{c_{i}}$ in the given factorization of the monodromy homeomorphism $\psi$. For simplicity, we will initially assume that the conjugating homeomorphism is a single Dehn twist, for example, $\bar{d} \mid$ and derive the result in this case. Recall for a circle $c_{i}$ that by axiom (Q3), we have $\overline{c_{i} \bar{d} \mid}=\left|d \overline{c_{i}}\right| \bar{d} \mid$, conjugation by $\bar{d} \mid$.

Thus if we initially have

$$
f_{a}\left(\left[c_{1}, \ldots, c_{n}\right]\right)=\left(a, c_{1}\right)+\left(a \overline{c_{1}} \mid, c_{2}\right)+\cdots+\left(a \overline{c_{1}}\left|\cdots \overline{c_{n-1}}\right|, c_{n}\right),
$$

then after conjugation by $\bar{d}$ we have the corresponding expression

$$
\begin{aligned}
& f_{a \bar{d} \mid}\left(\left[c_{1} \bar{d}\left|, \ldots, c_{n} \bar{d}\right|\right]\right)=\left(a \bar{d} \mid, c_{1} \bar{d}\right)+\left(a \bar{d} \overline{c_{1} \bar{d} \mid}, c_{2} \bar{d} \mid\right) \\
& \quad+\left(a \bar{d} \overline{c_{1} \bar{d} \mid} \overline{c_{2} \bar{d} \mid}, c_{3} \bar{d}\right)+\cdots+\left(a \bar{d} \overline{c_{1} \bar{d}}\left|\overline{c_{2} \bar{d}}\right| \ldots \overline{c_{n-1} \bar{d} \mid}, c_{n} \bar{d}\right),
\end{aligned}
$$

where $a \bar{d} \mid$ records the effect of the conjugating homeomorphism $\bar{d}$ on the "test circle" $a$. Now expanding the double brackets into conjugations, cancelling the adjacent $\bar{d} \mathrm{~s}$ and $\vec{d} \mid \mathrm{s}$, and applying the definition of the action by homeomorphisms on chains given in Section 2.2, we get

$$
\begin{aligned}
& f_{a d}\left(\left[c_{1} \bar{d}\left|, \ldots, c_{n} \bar{d}\right|\right]\right)=\left(a \bar{d}\left|, c_{1} \bar{d}\right|\right)+\left(a \overline{c_{1}}|\bar{d}|, c_{2} \bar{d} \mid\right)+\left(a \overline{c_{1}}\left|\overline{c_{2}}\right| \bar{d}\left|, c_{3} \bar{d}\right|\right) \\
& +\cdots+\left(a \overline{c_{1}}\left|\overline{c_{2}} \ldots \overline{c_{n-1}}\right| \bar{d}\left|, c_{n} \bar{d}\right|\right) \\
& =\left(a, c_{1}\right) \bar{d}+\left(a \overline{c_{1}}, c_{2}\right) \bar{d}+\left(a \overline{c_{1}} \mid \overline{c_{2}}, c_{3}\right) \bar{d} \\
& +\cdots+\left(a \overline{c_{1}}\left|\overline{c_{2}} \ldots \overline{c_{n-1}}\right|, c_{n}\right) \bar{d} \\
& =\left(f_{a}\left(\left[c_{1}, \ldots, c_{n}\right]\right)\right) \bar{d} \text {. }
\end{aligned}
$$


By the proof of Proposition 4.1 and the paragraph that follows it, cycles that differ by a Dehn twist are homologous. So the cycles corresponding under the appropriate maps to the original and conjugated monodromy $n$-tuples, $\left[c_{1}, \ldots, c_{n}\right]$ and $\left[c_{1} \bar{d}\left|, \ldots, c_{n} \bar{d}\right|\right.$, are homologous. If we now replace the single Dehn twist $\bar{d} \mid$ by a homeomorphism $\phi$ which is a product of Dehn twists, a similar argument goes through. So conjugate monodromy $n$-tuples yield homologous cycles.

We now look at the effect that applying an elementary transformation to a monodomy $n$-tuple has, on the corresponding quandle 2-cycles. From the description at the end of Section 5.1, taken from Fuller [3] or Gompf and Stipsicz [4], for a monodromy $n$-tuple having $b$ and $d$ as the $i$ th and $(i+1)$ st entries respectively, the elementary transformation corresponding to sliding the $(i+1)$ st arc, $\gamma_{i+1}$, over the $i$ th arc, $\gamma_{i}$, (see Section 5.1), has the following effect:

$$
[\ldots, b, d, \ldots] \longmapsto[\ldots, d, b \bar{d} \mid, \ldots] .
$$

Recall for the map $f_{x}$, the "test" circle $x$ becomes the first term of the first simplex in the image of a monodromy $n$-tuple. In the case at hand, this entry does not come into play, so the subscript $x$ will be suppressed, and the map will be written as $f$. Below, $a$ is just an arbitrary circle occurring in the $n$-tuple.

Let

$$
\chi_{1}=f([\ldots, b, d, \ldots])=\ldots+(a, b)+(c, d)+\ldots \quad \text { where } a \bar{b}=c
$$

and

$$
\chi_{2}=f([\ldots, d, b \bar{d} \mid, \ldots])=\cdots+(a, d)+(a \bar{d}|, b \bar{d}|)+\cdots
$$

be the image 2 -chains of the monodromy $n$-tuples. Since the elementary transformation only affects the $i$ th and $(i+1)$ st terms, all terms in $\chi_{1}$ except the pair shown are identical to terms in $\chi_{2}$, except for the pair shown. So forming the difference, we are left with

$$
\chi_{2}-\chi_{1}=(a, d)-(a, b)-(a \bar{b}, d)+(a \bar{d} \mid, b \bar{d})
$$

Now recall from the definition of the boundary operator on a 3-simplex, that

$$
\partial(a, b, d)=(a, d)-(a, b)-(a \bar{b} \mid, d)+(a \bar{d}|, b \bar{d}|),
$$

so $\chi_{2}-\chi_{1}=\partial(a, b, d)$. So positive cycles, corresponding to monodromy $n$-tuples which differ by an elementary transformation, are homologous. Thus statement (1) of the theorem is established. Visually, the effect of an elementary transformation can be realized by glueing in the appropriate square corresponding to the appropriate 3-simplex, with the notation and conventions described in Section 2.1. 
(2) We now address statement (2). If $\left[c_{1}, \ldots, c_{n}\right]$ corresponding to a homeomorphism $\psi$, is a monodromy $n$-tuple, let $F$ denote a regular fiber of the Lefschetz fibration over $D^{2}$, so $F \cong \Sigma_{g}$. The restriction of the fibration $\pi: X \rightarrow D^{2}$ over the boundary $S^{1}=\partial D^{2}$, is the mapping torus $S^{1} \times_{\psi} F$. If the circle $a \subset F$ is fixed by $\psi$, then $S^{1} \times a$ is a torus in $S^{1} \times_{\psi} F$. The pair $\left(\left[c_{1}, \ldots, c_{n}\right], a\right)$ uniquely determines such a torus in $\partial X$, and also clearly uniquely specifies the cycle $\chi$ under $f_{a}$. Conversely, given such a 2-cycle $\chi$, there is a unique way to order the simplices so that for all the simplices, the image of the first entry of a given simplex, under he Dehn twist specified by the second entry, is the first entry of the next simplex. The ordered list of second entries is the monodromy $n$-tuple, and the first entry in the first simplex is $a$. This then corresponds to a torus "anchored" at $a$. We now show such a torus is incompressible in $\partial X$. To do this, we shall make use of a well known result on incompressible surfaces in 3-manifolds. It appears in Hempel [5, Lemma 6.5] and Jaco [6, Lemma III.9]. Using this lemma, the fiber $F$, is the inverse image of a regular point $\star \in S^{1}$, and is incompressible in $S^{1} \times_{\psi} F$. We have the description given above in Section 5.1 of the boundary of Lefschetz fibered 4-manifold $X$, as

$$
\frac{\Sigma_{g} \times I}{(p, 0) \sim((p) \psi, 1)}
$$

and the space $X$ can be described as

$$
\left(\Sigma_{g} \times B\right) \bigcup_{i=1}^{n}(2-\text { handles }),
$$

where the 2-handles are attached along the vanishing cycles $c_{i}$ of the singular fibers. Thus we have

$$
\pi_{1}(X)=\frac{\pi_{1}\left(F \times D^{2}\right)}{\left\langle c_{1}, \ldots, c_{n}\right\rangle}=\frac{\pi_{1}(F)}{\left\langle c_{1}, \ldots, c_{n}\right\rangle},
$$

where $\left\langle c_{1}, \ldots, c_{n}\right\rangle$ is the subgroup generated by the classes of the $c_{i}$ 's in $F$.

Suppose the torus $S^{1} \times a$ was compressible in $\partial X$. It would then be compressible in $X$, so there would exist an essential loop $\lambda \subset S^{1} \times a$ which bounded an imbedded disk in $X$. So $\lambda \in\left\langle c_{1}, \ldots, c_{n}\right\rangle$. But then, some product of the loops $c_{1}, \ldots, c_{n}$ in $F$ is a loop bounding an imbedded disk. This contradicts the incompressibility of $F$ in $X$. So the torus $S^{1} \times a$ is incompressible in $\partial X$. This proves statement (2).

Remarks (1) Naïvely, the 2-homology classes just described provide obstructions to a fibration being a trivial fiber bundle over the base. The 0 homology class in $H_{2}(Q)$ corresponds to a fiber bundle with monodromy $=1 \in \operatorname{MCG}(F)$, having no singular fibers. Singular fibers, with nontrivial monodromy, clearly correspond to nonzero 2-cycles. 
(2) Consider Lefschetz fibrations over the disk $D^{2}$, with monodromy given by a relation in $\operatorname{MCG}(F)$, that is, where we may write $\psi=\overline{c_{1}}\left|\cdot \overline{c_{2}}\right| \cdots \overline{c_{n}}=$ $1_{\mathrm{MCG}(F)}$. Then since the monodromy is the identity on $\partial D^{2}$, we may glue on a second disk along $\partial D^{2}$, and extend the Lefschetz fibration to a Lefschetz fibration over $S^{2}$. Following Proposition 2.4, for the 2-cycles associated to a fibration with such monodromy, any circle a $\subset \Sigma_{g}$ is fixed by $\psi$, and could be used as the initial circle of such a 2-cycle. So Lefschetz fibrations over $S^{2}$ correspond to these "universal" 2-cycles.

(3) The families of relations of section 1, and their associated cycles from Section 2.4, (they are all purely positive Dehn twists) provide examples of possible monodromy homeomorphisms for Lefschetz fibrations over $D^{2}$, for instance with fibers $F$ of genus 2 or 3 . The resulting Lefschetz fibered manifolds have symplectic structures, and their boundaries exhibit the incompressible tori associated to the fixed circles, as specified in Theorem 5.3.

We end with the following:

Question 5.4 According to Theorem 5.3, isomorphic Lefschetz fibrations over $D^{2}$ correspond, for example, to homologous 2-cycles. Do the 3-chains bounded by pairs of such 2-cycles correspond to cobordisms between the fibrations, or to higher dimensional analogs of Lefschetz fibrations? If so, what is the correspondence, and can it be meaningfully generalized and interpreted with respect to higher dimensional cycles and boundaries?

Acknowledgments I would like to thank Profs. Carter, Przytycki, and Saito for interesting and useful conversations, and Prof. Przytycki for suggesting the $(-1)^{n+1}$ factor in the definition of $S_{n}^{a}$.

\section{References}

[1] J S Carter, S Kamada, M Saito, Geometric interpretations of quandle homology arXiv:math.GT/0006115

[2] R Fenn, C Rourke, Racks and links in codimension two, J. Knot Theory Ramifications 1 (1992) 343-406 MR1194995

[3] T Fuller, Lefschetz fibrations of 4-dimensional manifolds, Cubo Mat. Educ. 5 (2003) 275-294 MR2065735

[4] R E Gompf, A I Stipsicz, 4-manifolds and Kirby calculus, Graduate Studies in Mathematics 20, American Mathematical Society, Providence, RI (1999) MR1707327 
[5] J Hempel, 3-manifolds, AMS Chelsea Publishing, Providence, RI (2004) MR2098385

[6] W Jaco, Lectures on three-manifold topology, CBMS Regional Conference Series in Mathematics 43, American Mathematical Society, Providence, R.I. (1980) MR565450

[7] L H Kauffman, Knots and physics, second edition, World Scientific Publishing Co., River Edge, NJ (1993) MR1306280

[8] W B R Lickorish, An Introduction to Knot Theory, Graduate Texts in Mathematics 175, Springer, New York (1997) MR1472978

[9] J Zablow, Loops and disks in surfaces and handlebodies, J. Knot Theory Ramifications 12 (2003) 203-223 MR1967241

Department of Mathematics, Rochester Institute of Technology

85 Lomb Memorial Drive, Rochester NY 14623, USA

dquandle@netscape.net

Received: 4 October 2007 